\title{
Characterisation and discrimination of various types of lac resin using gas chromatography mass spectrometry techniques with quaternary ammonium reagents
}

\author{
K. Sutherland ${ }^{\mathrm{a}, *}$, J.C. del Río ${ }^{\mathrm{b}}$ \\ a Philadelphia Museum of Art, Conservation Department, Box 7646, Philadelphia, PA 19101, USA \\ b Institute for Natural Resources and Agrobiology, Department of Plant Biotechnology, Box 1052, 41080 Seville, Spain
}

\section{A R T I C L E I N F O}

\section{Article history:}

Received 9 December 2013

Received in revised form 4 February 2014

Accepted 20 February 2014

Available online 28 February 2014

\section{Keywords:}

Lac resin

Shellac

Hydroxyacids

GCMS

Quaternary ammonium reagents

Museum artefacts

\begin{abstract}
A B S T R A C T
A variety of lac resin samples obtained from artists' suppliers, industrial manufacturers, and museum collections were analysed using gas chromatography mass spectrometry (GCMS) and reactive pyrolysis GCMS with quaternary ammonium reagents. These techniques allowed a detailed chemical characterisation of microgram-sized samples, based on the detection and identification of derivatives of the hydroxy aliphatic and cyclic (sesquiterpene) acids that compose the resin. Differences in composition could be related to the nature of the resin, e.g. wax-containing (unrefined), bleached, or aged samples. Furthermore, differences in the relative abundances of aliphatic hydroxyacids appear to be associated with the biological source of the resin. The diagnostic value of newly characterised lac components, including 8hydroxyacids, is discussed here for the first time. Identification of derivatised components was aided by AMDIS deconvolution software, and discrimination of samples was enhanced by statistical evaluation of data using principal component analysis. The robustness of the analyses, together with the minimal sample size required, make these very powerful approaches for the characterisation of lac resin in museum objects. The value of such analyses for enhancing the understanding of museum collections is illustrated by two case studies of objects in the collection of the Philadelphia Museum of Art: a restorer's varnish on a painting by Luca Signorelli, and a pictorial inlay in an early nineteenth-century High Chest by George Dyer.
\end{abstract} (c) 2014 Elsevier B.V. All rights reserved.

\section{Background}

\subsection{Origin, varieties and uses of lac resin}

Lac resin is the secretion of scale insects (Kerria lacca, Kerria chinensis, and related species) cultivated mainly in India, Thailand and other parts of Southeast Asia [1-3]. The material forms a dense encrustation on the branches of host plants infested by the insects, and when harvested in this form it is referred to as stick-lac. After crushing and washing in water to remove plant matter, insect remains, and a red colourant (lac dye), the semi-refined material is known as seedlac. Further processing involves heating and filtering the seedlac through a cloth or wire mesh, or solvent extraction, to produce the various grades of commercial resin. This is supplied in the form of small medallions (button lac) or, more

\footnotetext{
* Corresponding author at: Art Institute of Chicago, Conservation Department, 111 South Michigan Avenue, Chicago, IL 60603, USA. Tel.: +1 3124437258 ; fax: +1 3125411959 .

E-mail address: ksutherland@artic.edu (K. Sutherland).
}

commonly, stretched or spread into thin sheets and broken into small fragments; in this latter form the resin is known as shellac. Various grades of shellac are produced, categorised mainly according to their colour - e.g. garnet, orange, lemon and blonde - or with reference to the strain of insect or season of harvest (kusmi, bysakhi, etc.) Additional processing can include chemical (hypochlorite) bleaching, to produce a highly transparent grade of resin known as bleached or "white" shellac, and/or refining to remove a naturally occurring wax component. More detailed discussions of lac resin and its processing can be found in the literature [4-6].

Although the major commercial markets for shellac are for architectural applications (wood finishes) and coatings for pharmaceuticals and food [7], this paper is concerned with its study in works of art and cultural artefacts. Lac resin is of great historical interest because of its use for centuries for a variety of purposes such as an adhesive, coating (varnish) or decorative inlay material. A precise characterisation of the resin in museum objects can provide information on artistic and restoration practices, shed light on alteration and deterioration processes, and inform safe and effective treatments essential for their preservation. 


\subsection{Composition and analysis}

The chemistry of lac resin is complex and not fully understood, although several published reviews provide a useful overview $[6,8]$. The dye and wax components of the raw material combined make up c. $10-15 \%$ of the total. The major, resinous component is a mixture of mono- and polyesters formed from hydroxy aliphatic acids, primarily 9,10,16-trihydroxy palmitic or aleuritic acid, linked with cyclic sesquiterpene acids of various structures. A major sesquiterpene in the fresh resin is jalaric acid, a monocarboxylic acid with one aldehyde and two hydroxy groups, along with a smaller proportion of laccijalaric acid, a related compound with a single hydroxy group. The resin fraction has been described in terms of 'hard' (ether-insoluble) and 'soft' (ether-soluble) resin components, along with additional sub-divisions, although these categories are somewhat arbitrary and differentiated mainly on the basis of the molecular weight of the ester oligomers. Fig. 1 shows the approximate (average) structure for 'pure lac resin', a fraction isolated from the hard resin, comprising four molecules each of the aleuritic and terpene acids [6,9], along with structures of the principal hydroxyacids.

A number of analytical techniques have been applied to the study of lac resin, with spectroscopic methods most widely used in the cultural heritage field. Fourier transform infrared spectroscopy (FTIR) is well-established [10], and complementary techniques such as far-IR, Raman and fluorescence spectroscopy have been explored more recently [11-14]. Although spectroscopy can be useful to indicate the presence of lac resin, it is not generally reliable for the specific identification of natural resins when aged and/or present in mixtures, as is often the case with samples obtained from museum objects [15]. Because of the unique chemistry of lac resin - as compared to other resinous materials derived from plants (di- and triterpenes) or modern synthetic polymers - the most effective and definitive methods for its identification in complex, microscopic-sized samples are based on mass spectrometry and chromatography. Some promise has been shown by the use of matrix- and graphite-assisted laser desorption ionisation mass spectrometry (MALDI, GALDI) $[16,17]$, but the most detailed compositional information has been provided by GCMS and Py-GCMS, which allow the separation and identification of the characteristic hydroxy aliphatic and terpene acids (and in waxcontaining grades, the wax components also). Several protocols for the analysis of lac resin have been published, including the use of Py-GCMS without derivatisation [18]: the value of this approach

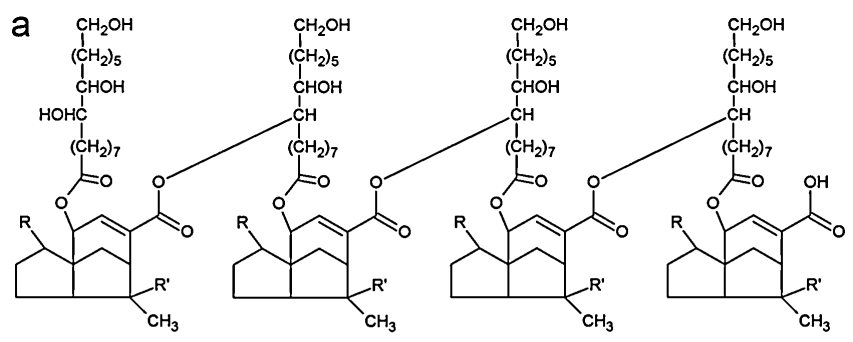

b

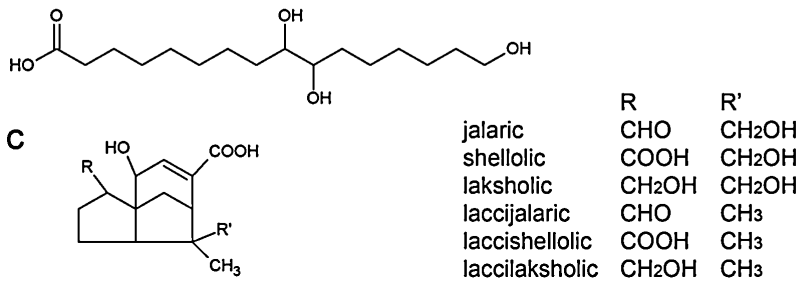

Fig. 1. Generalised structure of "pure lac resin" (a), with structures for aleuritic acid (b) and cyclic terpene acids (c) that are the major compounds obtained by hydrolysis of the resin esters. is limited because of the numerous hydroxy and carboxylic acid groups in the lac constituents, however, and more reliable characterisations have been achieved by the use of silylating [19] or methylating reagents [20-22] to produce derivatives with improved chromatographic properties. In this study both GCMS and Py-GCMS were used with quaternary ammonium methylating reagents: ( $m$-trifluoromethylphenyl)trimethylammonium hydroxide (also known as trimethyl[ $\alpha, \alpha, \alpha$-trifluoro- $m$-tolyl $]$ ammonium hydroxide or TMTFTH, and available commercially as 'MethPrep II' [23]) for GCMS, and tetramethylammonium hydroxide (TMAH) for Py-GCMS.

TMTFTH and TMAH have gained widespread use for the study of organic materials in works of art since they give efficient derivatisation without complicated sample pretreatment or extraction steps - an important consideration for samples that are often very small and complex [23-32]. Whereas TMAH is typically used with a pyrolysis interface, TMTFTH has been shown to be effective at lower temperatures and can therefore be used readily without a pyrolyser, by injection of a sample/reagent solution directly into a conventional GC inlet. TMTFTH is also favoured by some researchers since it has a lower alkalinity and avoids some undesirable side-reactions, such as isomerisation of polyunsaturated fatty acids, that can occur with TMAH [23,24,29,31,32]. The use of TMAH with Py-GCMS is commonly termed 'reactive' PyGCMS or thermally assisted hydrolysis and methylation Py-GCMS (THM-Py-GCMS). Both reagents act by promoting the hydrolysis of the resin (poly)esters and methylation of free acid and (to some extent) alcohol groups to produce volatile methyl esters and ethers $[23,24,33]$. A complication of TMTFTH is that it can produce (trifluoromethyl)phenyl (TFMP) ethers, in addition to methyl ethers, upon reaction with certain alcohols $[34,35]$ : although these compounds are essentially artefacts and complicate the interpretation of data, as discussed below, they have the serendipitous advantage of producing mass spectra that are distinctive and diagnostic, and thus have value as chemical markers.

This paper presents comparative data from the analysis of a wide variety of reference samples of lac resin from commercial artists' suppliers, industrial manufacturers, and museum collections using the two analytical approaches. Aspects of this research dealing specifically with bleached shellac and its use as a varnish for paintings have been described in separate papers $[21,22]$. Whereas some previous research has treated shellac as a generic material, without attempting to discriminate different varieties, this study represents a significant advance in the characterisation of the resin, highlighting the potential to differentiate lac resins of different grades, ages and biological/geographical sources. Discrimination is enhanced by statistical evaluation of the (Py)GCMS data using principal component analysis. Mass spectra for selected diagnostic derivatives, most of which are previously unpublished, are provided in an Appendix. The value of the analytical approaches is illustrated by two case studies of objects in the collection of the Philadelphia Museum of Art (PMA): a restoration varnish on a painting by Luca Signorelli (c.1445-1523) and a pictorial inlay in an early nineteenth-century High Chest by George Dyer (active 1805-46).

\section{Experimental}

\subsection{Samples}

45 samples of lac resin, representing a range of characteristics such as grade, age, and geographical origin, were obtained for analysis as listed in Table 1 . Aleuritic acid was supplied by Sigma-Aldrich, St. Louis, MO 63178, USA. Samples of varnish from the Signorelli painting and inlay material from the Dyer High Chest 
Table 1

Lac resin reference materials analysed; all are dry, bulk resins and recently acquired/manufactured unless otherwise specified.

\begin{tabular}{|c|c|c|c|}
\hline Sample & Description & Source & Comments \\
\hline 1 & Stick lac, Siam (Thailand) & Commercial Museum, Philadelphia & Late 19th/early 20th C \\
\hline 2 & Seedlac & A. F. Suter \& Co. Ltd. & \\
\hline 3 & Seedlac & Behlen & \\
\hline 4 & Seedlac & Kremer Pigmente & \\
\hline 5 & Seedlac, Thai & Shellac.net & \\
\hline 6 & Seedlac, Indian, Kusmi & Shellac.net & \\
\hline 7 & Seedlac, Indian, Bysakhi & Shellac.net & \\
\hline 8 & Seedlac, Thai & Gifu Shellac Manufacturing Co. Ltd. & \\
\hline 9 & Seedlac, Indian & Gifu Shellac Manufacturing Co. Ltd. & \\
\hline 10 & Button lac & A. F. Suter \& Co. Ltd. & \\
\hline 11 & Button lac, Indian & Agra, India & \\
\hline 12 & Button lac, Indian, Bysakhi & Shellac.net & \\
\hline 13 & Button lac, Indian, Kusmi & Shellac.net & \\
\hline 14 & Garnet shellac & A. F. Suter \& Co. Ltd. & \\
\hline 15 & Garnet shellac, AC & Behlen & \\
\hline 16 & Garnet shellac & J. E. Moser's & \\
\hline 17 & Shellac, Thai, GS & Gifu Shellac Manufacturing Co. Ltd. & \\
\hline 18 & Shellac, dark orange & Commercial Museum, Philadelphia & Late 19 th/early 20 th C \\
\hline 19 & Orange shellac & Behlen & \\
\hline 20 & Orange shellac & Gettens Materials Collection & Bulk material, early 20th C \\
\hline 21 & Orange shellac & Gettens Materials Collection & From thin film cast 1932 \\
\hline 22 & Orange shellac & Michael McNamara Varnish Works & Dried residue from ceramic bottle; late 19th/early 20th C? \\
\hline 23 & Yellow shellac & Kremer Pigmente & \\
\hline 24 & Lemon shellac & A. F. Suter \& Co. Ltd. & \\
\hline 25 & Lemon shellac \#1 & Mantrose-Haeuser Co., Inc. & \\
\hline 26 & Lemon shellac, Indian & Gifu Shellac Manufacturing Co. Ltd. & \\
\hline 27 & Blonde shellac & Mohawk Finishing Products & \\
\hline 28 & Blonde shellac & Angelo Bros. & Age unknown \\
\hline 29 & Super blonde shellac & Behlen & \\
\hline 30 & Bleached shellac very light & Kremer Pigmente & Decolourised with charcoal, not chemically bleached \\
\hline 31 & Bleached shellac, regular & Temuss Products Ltd. & \\
\hline 32 & Bleached shellac, refined & Temuss Products Ltd. & \\
\hline 33 & White shellac & W. S. Jenkins \& Co. Ltd. & \\
\hline 34 & Transparent shellac & W. S. Jenkins \& Co. Ltd. & \\
\hline 35 & Bleached shellac & Wood Finishing Enterprises & \\
\hline 36 & Bleached shellac, R52 & Mantrose-Haeuser Co., Inc. & \\
\hline 37 & Bleached shellac, R49 & Mantrose-Haeuser Co., Inc. & \\
\hline 38 & White shellac & Victoria and Albert Museum, London & Age unknown \\
\hline 39 & Bleached shellac & Doerner Institut, Munich & Early sample, exact date unknown \\
\hline 40 & Bleached shellac & National Museum of African Art, Washington DC & From thin film cast 1992 \\
\hline 41 & Refined shellac & National Museum of African Art, Washington DC & From thin film cast 1992 \\
\hline 42 & White shellac & Gettens Materials Collection & From thin film cast 1933 \\
\hline 43 & Pure white shellac, Bulls Eye Brand & Florence Griswold Museum, CT & Dried residue from jar; early 20th C? \\
\hline 44 & Pure white shellac, Derayco & Florence Griswold Museum, CT & Liquid (ethanol solution?); early 20th C \\
\hline 45 & Soehnée Frères retouching varnish & Florence Griswold Museum, CT & Liquid (ethanol solution?); early 20th C \\
\hline
\end{tabular}

were provided by PMA conservators Allen Kosanovich and Peggy Olley, respectively.

\subsection{GCMS}

Samples (c. 10-50 $\mu \mathrm{g}$ ) were prepared for GCMS analysis by adding a 1:1 mixture of benzene and MethPrep II (Alltech, 5 wt.\% in methanol), and left to react overnight at room temperature. The reaction vial was centrifuged, and $1 \mu \mathrm{L}$ of the sample solution was injected into the GC. An Agilent 6890N GC was used with a J\&W DB-5MS column ( $30 \mathrm{~m}, 0.25 \mathrm{~mm}$ i.d., $0.25 \mu \mathrm{m}$ film), interfaced to a $5973 \mathrm{~N}$ MS. The GC inlet temperature was $300^{\circ} \mathrm{C}$, MS interface $300^{\circ} \mathrm{C}$. The oven was programmed from $50^{\circ} \mathrm{C}$, with a 2 min hold, increased at $10^{\circ} \mathrm{Cmin}^{-1}$ to $310^{\circ} \mathrm{C}$, and held isothermally for $12 \mathrm{~min}$; total run time $40 \mathrm{~min}$. The inlet was operated in splitless mode, with 0.7 min purge-on time. Helium was the carrier gas, with a linear velocity of $45 \mathrm{~cm} / \mathrm{s}$. The MS was run in scan mode $(\mathrm{m} / \mathrm{z}$ 50-750) with the source at $230^{\circ} \mathrm{C}$ and the quadrupole at $150^{\circ} \mathrm{C}$. Data were processed using Agilent Chemstation software; AMDIS (Automated Mass Spectral Deconvolution and Identification System, NIST) was used in some cases for deconvolution of mass spectra. Detected compounds were identified from interpretation of their electron ionisation (EI) mass spectra, with reference to NIST05 and Wiley275 databases and published literature; and from retention times $\left(t_{R}\right)$, in particular the relative $t_{R}$ of analogous compounds with different numbers and types of substituent (e.g. hydroxy, methoxy or trifluoromethylphenoxy, as discussed below).

\subsection{THM-Py-GCMS}

Samples (c. 10-50 $\mu \mathrm{g}$ ) were placed in Frontier Lab stainless steel sample cups for analysis by THM-Py-GCMS. $2 \mu \mathrm{L}$ of a $25 \mathrm{wt} . \%$ solution of TMAH in methanol, or a $10 \mathrm{wt} . \%$ aqueous solution (both reagents Sigma-Aldrich), was added and the solvent evaporated prior to insertion into a Frontier PY-2020iD vertical microfurnace pyrolyser, attached to the Agilent 6890N GC. The furnace temperature was $550^{\circ} \mathrm{C}$ for most analyses. Other parameters were as above, with the following exceptions: the GC oven was programmed from $40^{\circ} \mathrm{C}$, with a $2 \mathrm{~min}$ hold, then increased at $20^{\circ} \mathrm{C} \mathrm{min}^{-1}$ to $140^{\circ} \mathrm{C}$, at $15^{\circ} \mathrm{C} \mathrm{min}{ }^{-1}$ to $320^{\circ} \mathrm{C}$, and held isothermally for $11 \mathrm{~min}$; total run time $30 \mathrm{~min}$. The inlet was operated with a split ratio of 1:10. Helium was the carrier gas, with a flow of $1 \mathrm{ml} / \mathrm{min}$. MS interface temperature was $320^{\circ} \mathrm{C}$; the MS was run in scan mode $(\mathrm{m} / \mathrm{z}$ 35-600). 


\section{4. $P C A$}

Principal component analysis (PCA) of (Py)GCMS data was carried out using OriginPro 8.6 software. The data matrix for PCA consisted of integrated areas of twelve selected chromatographic peaks that could be identified as methyl derivatives of characteristic hydroxy aliphatic and terpene acids (see Section 3.2; one unidentified compound that has been cited as a marker compound in studies of shellac was also included in the analysis). Peak areas were integrated from selected ion chromatograms corresponding to characteristic ions in the mass spectra of each compound, rather than from the TIC, to minimise problems with quantitation of coeluting peaks. For TMAH analyses, data from 45 reference samples were included, along with the Signorelli varnish sample. Preprocessing of data was found to strongly influence the PCA results: various approaches were tested, based on recommendations in the literature for chromatographic and mass spectral data [36,37]. The results presented in this paper were generated using a $\log _{10}$ transformation of the integrated values, to minimise discrepancies relating to sample size, the relative abundance of components, and the use of different quantitation ions; followed by PCA of the covariance matrix. For TMTFTH data, obtained from a subset of 19 reference samples, an added step of row-scaling (i.e. scaling the integrated peak areas for each sample to a constant total) prior to log transformation was found to be advantageous, presumably because of the greater variation in chromatographic response for these analyses.

\section{Results and discussion}

\subsection{Analysis of lac reference samples with TMAH and TMTFTH}

Total ion chromatograms (TICs) from THM-Py-GCMS and GCMS analysis of two lac resin samples - a shellac of Thai origin (Gifu Company) and an orange shellac (origin unknown, Gettens Materials Collection) - are shown in Fig. 2. Both techniques proved effective for the derivatisation and separation of the characteristic aliphatic and terpene hydroxyacids in the lac resins; the identified compounds are listed in Table 2 and mass spectra for key diagnostic compounds are provided in Appendix. The results obtained with TMAH are generally in agreement with the findings of Wang et al. [20], supplemented with the identification for the first time of several novel diagnostic compounds, as discussed below; the results for GCMS with TMTFTH are also described here for the first time.

Multiple derivatives were observed for individual hydroxy compounds using TMAH (Fig. 2a and b), primarily a consequence of partial methylation of hydroxyl groups by the reagent, an effect that has been observed in previous studies using this reagent $[20,33,38,39]$. Multiple analyses of individual samples showed good repeatability and the relative abundance of derivatives with different degrees of methylation was found to be fairly consistent from sample to sample, although dependent to some extent on factors such as sample size. For terpene components, the presence of additional peaks in some cases is a consequence of the occurrence of these compounds as stereoisomers (epimers $[6,19]){ }^{1}$

The major peak for all samples analysed with TMAH was the permethylated (methyl ester, trimethyl ether) form of aleuritic acid (\#28), with another peak of variable intensity corresponding to coeluting isomers of the trimethylated (methyl ester, dimethyl ether) form of this compound (\#29,30). An additional aleuritic acid

\footnotetext{
1 Since terpene epimers could not be distinguished from their EI mass spectra, terms for the specific isomers are not used in this paper: e.g. "shellolic acid" is used to refer either to shellolic or epishellolic acid, the latter being the more common naturally occurring isomer.
}

derivative (\#27) was not conclusively identified but its mass spectrum (including a prominent peak at $m / z 155$ ) suggests that it may result from dehydration in the pyrolyser prior to methylation. Peaks eluting earlier in the TIC correspond to derivatives of other aliphatic and terpene acids, as listed in Table 2. Some of the chromatographic peaks were found to comprise several related compounds that were not separated under the conditions used: for example, 8-hydroxy myristic acid $(\# 3,6)$ was often found along with smaller amounts of the 7- and 9-hydroxy compounds (see Table 2). To the best of authors' knowledge these compounds have not been reported previously in chromatographic studies of shellac. In general, the overall pattern of peaks observed was similar for the samples studied although variations were observed in the relative proportions of certain aliphatic and terpene species that could be related to the nature of the sample, as discussed in more detail below.

In a previous study of lac resin using THM-Py-GCMS, Wang and co-workers recommended the use of TMAH in aqueous solution rather than in methanol, and a relatively low furnace temperature of $400^{\circ} \mathrm{C}$ to minimise pyrolytic degradation of the characteristic resin components [20]. In the current study, initial analyses of resin samples using aqueous and methanolic TMAH solutions showed some variation in the distribution of the products obtained: for example, a higher yield of terpene derivatives was observed using aqueous TMAH in some cases, and a lower yield of the fatty alcohol methyl ethers. In general, however, the differences were not pronounced, and the more volatile methanolic TMAH solution was used for most analyses for convenience (i.e. for the more rapid drying of the reagent solution prior to analysis). Different pyrolyser temperatures $\left(350,400\right.$ and $\left.550^{\circ} \mathrm{C}\right)$ were also tested and did not show substantial variations in the distribution of products; the higher temperature was generally used since a versatile method was sought that would allow for the analysis of a wide range of materials that might be encountered in a complex sample from a work of art.

A concern that has been expressed for the analysis of lac resin using alkaline reagents such as TMAH is the possibility of disproportionation of the aldehyde-containing terpenes (jalaric and laccijalaric acids) via the Cannizzaro reaction to produce corresponding compounds in which the aldehyde group is replaced by an acid (shellolic and laccishellolic acids) and an alcohol (laksholic and laccilaksholic acids; see Fig. 1), with the resultant compounds converted to their methyl esters and ethers [20]. This reaction would complicate interpretation of the data since the resulting compounds are also present naturally in the resin [19]. Cannizzaro disproportionation in the presence of TMAH has been demonstrated previously only for aromatic aldehydes, which lack a hydrogen in the $\alpha$-position and are typically more susceptible to this reaction $[40,41]$. Aldol and retroaldol reactions have been observed with other types of aldehyde, such as simple carbohydrates [42], but these appear less likely for the lac terpenes because of steric hindrance [19]. In the current study these phenomena did not appear to play a significant role. As discussed below, the relative proportions of (lacci)jalaric and (lacci)shellolic acids appear primarily to reflect oxidation relating to the age and processing of the resin rather than to derivatisation effects.

Interestingly, jalaric and laccijalaric acids were detected mainly as their tetra- and trimethylated derivatives, respectively (\#9,12,18,22), suggesting methylation of the aldehyde in addition to the acid and alcohol groups. Wang et al. reported that the products were methyl ketones, although a mechanism was not proposed [20; see also 43]. This methylation seems unlikely since the aldehyde group is not acidic and would not be expected to undergo reaction with TMAH in the usual way, via deprotonation and formation of a tetramethylammonium salt [23]. Other studies have reported methylation by TMAH of the enol tautomer of carbonyl 

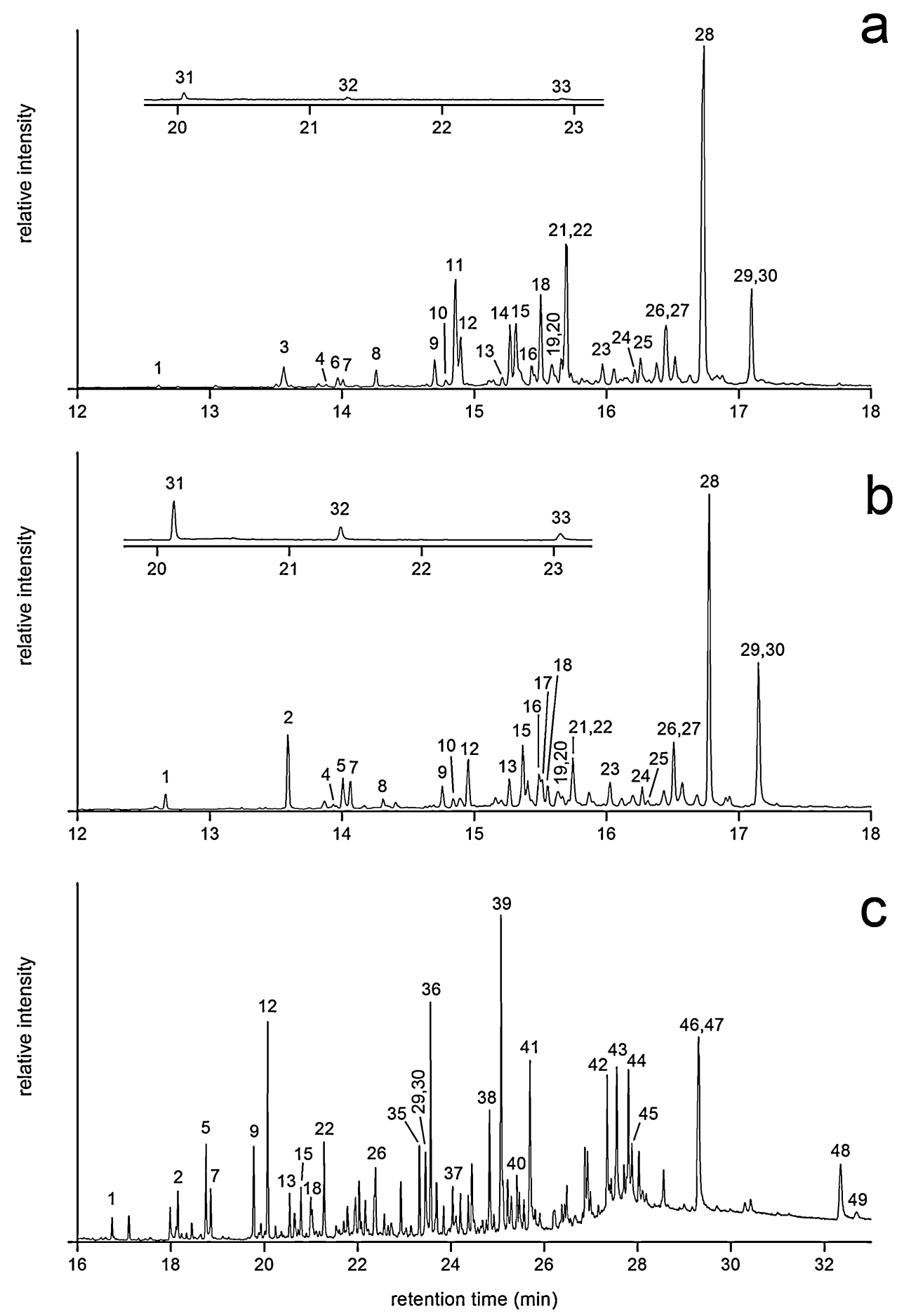

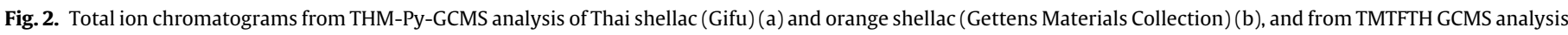

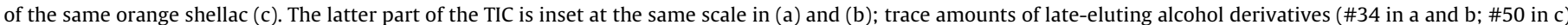
are not shown. Peak labels refer to Table 2.

groups in di- and triterpenes, to produce a methyl ether with adjacent $\mathrm{C}=\mathrm{C}$ unsaturation $[26,28]$. Methylation can also occur on reactive carbons adjacent to certain functional groups in terpenes and fatty acids $[26,44]$, and a similar reaction may be plausible for the jalaric and laccijalaric acids. Further study is required to elucidate the reactions; in this paper the products are referred to as "methyl derivatives" without assigning a specific structure to the methylated aldehyde.

In addition to the methylated acids derived from hydrolysis of esters, the TMAH data show fatty alcohols corresponding to the naturally occurring wax component of lac resin. The alcohols, primarily C28 (octacosanol) with smaller amounts of C30, C32 and C34 [45], are detected as their methyl ethers.

Analysis using GCMS with TMTFTH also resulted in a characteristic pattern of derivatised aliphatic and terpene acids, as shown for the Gettens orange shellac in Fig. 2c. The TICs were more complex than those obtained using TMAH, a consequence of reactions undergone by alcohols with TMTFTH to produce TFMP in addition to methyl ethers [35]. Because of the multiple derivatives produced from polyhydroxy compounds with this reagent (methyl, TFMP and mixed ethers), peak assignment for the lac samples is challenging; tentative identifications for TFMP 
Table 2

Detected products of derivatisation using TMAH and TMTFTH.

\begin{tabular}{|c|c|c|c|c|c|}
\hline Label & $t_{\mathrm{R}} 1^{\mathrm{a}}$ & $t_{\mathrm{R}} 2^{\mathrm{b}}$ & Compound & MW & Base peak \\
\hline 1 & 12.61 & 16.74 & Myristic acid: methyl ester & 242 & 74 \\
\hline 2 & 13.54 & 18.15 & Butolic acid: methyl ester, methyl ether & 272 & 127 \\
\hline 3 & 13.56 & 18.19 & 8-Hydroxymyristic acid: methyl ester, methyl ether ${ }^{c}$ & 272 & 187 \\
\hline 4 & 13.88 & 18.65 & Hexadecenoic acid: methyl ester ${ }^{\mathrm{d}}$ & 268 & 55 \\
\hline 5 & 13.95 & 18.75 & Butolic acid: methyl ester & 258 & 87 \\
\hline 6 & 13.97 & 18.77 & 8-Hydroxymyristic acid: methyl ester ${ }^{e}$ & 258 & 87 \\
\hline 7 & 14.01 & 18.85 & Palmitic acid: methyl ester & 270 & 74 \\
\hline 8 & 14.26 & 19.24 & 9,10-Dihydroxymyristic acid: methyl ester, dimethyl ether & 302 & 201 \\
\hline 9 & 14.70 & 19.78 & Laccijalaric acid: trimethyl derivative (isomer of \#12) & 306 & 306 \\
\hline 10 & 14.79 & 19.89 & Laccilaksholic acid: methyl ester, dimethyl ether & 308 & 276 \\
\hline 11 & 14.86 & 20.13 & 8-Hydroxypalmitic acid: methyl ester, methyl ether ${ }^{\mathrm{f}}$ & 300 & 187 \\
\hline 12 & 14.90 & 20.07 & Laccijalaric acid: trimethyl derivative (isomer of \#9) & 306 & 306 \\
\hline 13 & 15.21 & 20.55 & Laccishellolic acid: dimethyl ester, methyl ether & 322 & 262 \\
\hline 14 & 15.27 & 20.73 & 8-Hydroxypalmitic acid: methyl ester ${ }^{g}$ & 286 & 141 \\
\hline 15 & 15.31 & 20.79 & 16-Hydroxyhexadecenoic acid: methyl ester, methyl ether ${ }^{d}$ & 298 & 67 \\
\hline 16 & 15.43 & 21.01 & 9,10-Dihydroxypalmitic acid: methyl ester, dimethyl ether & 330 & 201 \\
\hline 17 & 15.46 & 21.03 & 16-Hydroxypalmitic acid: methyl ester, methyl ether & 300 & 74 \\
\hline 18 & 15.50 & 21.00 & Jalaric acid: tetramethyl derivative (isomer of \#22) & 336 & 291 \\
\hline 19 & 15.58 & n.d. ${ }^{\mathrm{h}}$ & Laksholic acid: methyl ester, trimethyl ether & 338 & 306 \\
\hline 20 & 15.62 & 21.11 & Unknown & & 247 \\
\hline 21 & 15.68 & 21.20 & Unknown & & 247 \\
\hline 22 & 15.70 & 21.28 & Jalaric acid: tetramethyl derivative (isomer of \#18) & 336 & 291 \\
\hline 23 & 15.97 & 21.70 & Shellolic acid: dimethyl ester, dimethyl ether & 352 & 320 \\
\hline 24 & 16.22 & n.d. & 9,16-Dihydroxypalmitic acid: methyl ester, dimethyl ether & 330 & 201 \\
\hline 25 & 16.26 & 22.08 & Jalaric acid: trimethyl derivative (isomer of \#26) & 322 & 291 \\
\hline 26 & 16.44 & 22.36 & Jalaric acid: trimethyl derivative (isomer of \#25) & 322 & 291 \\
\hline 27 & 16.46 & 22.48 & Aleuritic acid: dehydrated derivative? & & 45 \\
\hline 28 & 16.72 & 22.93 & Aleuritic acid: methyl ester, trimethyl ether & 360 & 71 \\
\hline 29 & 17.09 & 23.45 & Aleuritic acid: methyl ester, 10,16-dimethyl ether & 346 & 95 \\
\hline 30 & 17.10 & 23.46 & Aleuritic acid: methyl ester, 9,16-dimethyl ether & 346 & 145 \\
\hline 31 & 20.05 & 27.77 & 1-Octacosanol: methyl ether & 424 & 83 \\
\hline 32 & 21.29 & n.d. & 1-Triacontanol: methyl ether & 452 & 57 \\
\hline 33 & 22.91 & n.d. & 1-Dotriacontanol: methyl ether & 480 & 57 \\
\hline 34 & 25.15 & n.d. & 1-Tetratriacontanol: methyl ether & 508 & 57 \\
\hline 35 & & 23.33 & Laccijalaric acid: dimethyl, TFMP ether (isomer of \#36) & 436 & 179 \\
\hline 36 & & 23.56 & Laccijalaric acid: dimethyl, TFMP ether (isomer of \#35) & 436 & 179 \\
\hline 37 & & 24.04 & Laccishellolic acid: dimethyl ester, TFMP ether & 452 & 231 \\
\hline 38 & & 24.83 & Jalaric acid: trimethyl, TFMP ether (isomer of \#39) & 466 & 291 \\
\hline 39 & & 25.07 & Jalaric acid: trimethyl, TFMP ether (isomer of \#38) & 466 & 291 \\
\hline 40 & & 25.41 & Shellolic acid: dimethyl ester, methyl ether, TFMP ether & 482 & 229 \\
\hline 41 & & 25.69 & Aleuritic acid: methyl ester, dimethyl ether, TFMP ether ${ }^{\mathrm{i}}$ & 490 & \\
\hline 42 & & 27.35 & Jalaric acid: dimethyl, di-TFMP ether (isomer of \#43) & 596 & 205 \\
\hline 43 & & 27.55 & Jalaric acid: dimethyl, di-TFMP ether (isomer of \#42) & 596 & 205 \\
\hline 44 & & 27.80 & Aleuritic acid: methyl ester, methyl ether, di-TFMP ether & 620 & 95 \\
\hline 45 & & 27.88 & Shellolic acid: dimethyl ester, di-TFMP ether & 612 & 229 \\
\hline 46 & & 29.29 & Aleuritic acid: methyl ester, 9-methyl ether, 10,16-di-TFMP ether & 620 & 201 \\
\hline 47 & & 29.31 & Aleuritic acid: methyl ester, 10-methyl ether, 9,16-di-TFMP ether & 620 & 289 \\
\hline 48 & & 32.35 & Aleuritic acid: methyl ester, tri-TFMP ether ${ }^{\mathrm{j}}$ & 750 & 95 \\
\hline 49 & & 32.69 & 1-Octacosanol: TFMP ether & 554 & 57 \\
\hline 50 & & 35.94 & 1-Triacontanol: TFMP ether & 582 & 57 \\
\hline
\end{tabular}

${ }^{a}$ Retention time using THM-Py-GCMS parameters (see Section 2).

b Retention time using GCMS parameters (see Section 2).

c Typically found co-eluting with 7-hydroxy (ions at $\mathrm{m} / \mathrm{z} 143$ and 173) and 9-hydroxy (ions at $\mathrm{m} / \mathrm{z} 115$ and 201 ) isomers.

d Position of double bond could not be determined from mass spectrum.

e Typically found co-eluting with 7-hydroxy (ions at $\mathrm{m} / \mathrm{z} 127$ and 159) and 9-hydroxy (ions at $\mathrm{m} / \mathrm{z} 155$ and 187 ) isomers.

f Typically found co-eluting with 9-hydroxy (ions at $\mathrm{m} / \mathrm{z} 143$ and 201) and other isomers.

$g$ Typically found co-eluting with 9-hydroxy (ions at $m / z 155$ and 187) and other isomers.

h Not detected.

i Several isomers co-eluting.

j Possibly with unidentified co-eluting compounds.

derivatives of a number of characteristic compounds are listed in Table 2, following the methyl derivatives. Fatty alcohols were observed, primarily as their TFMP ethers, late in the run (after $32 \mathrm{~min}$ ).

Mass spectra for the derivatised compounds show certain consistent features that facilitate their recognition in complex samples (see Appendix). The methylated terpene acids are typically characterised by a series of peaks including $[\mathrm{M}-15]^{+},[\mathrm{M}-31]^{+},[\mathrm{M}-32]^{+}$, $[\mathrm{M}-45]^{+},[\mathrm{M}-47]^{+},[\mathrm{M}-60]^{+}$and/or $[\mathrm{M}-64]^{+}$, corresponding to cleavage of side-groups (such as methyl and methoxy groups, and combinations of these) from the cyclic molecule; along with the molecular ion [21]. For the TFMP derivatives, additional peaks are often found at $[\mathrm{M}-161]^{+}$or $[\mathrm{M}-162]^{+}$corresponding to loss of $\mathrm{CF}_{3} \mathrm{C}_{6} \mathrm{H}_{4} \mathrm{O}$ or $\mathrm{CF}_{3} \mathrm{C}_{6} \mathrm{H}_{4} \mathrm{OH}$ [35], and in combination with the other losses described above. In mass spectra for the methylated aliphatic hydroxyacids the molecular ion is small or absent; a small peak at $[\mathrm{M}-31]^{+}$may be present and other characteristic ions include those resulting from cleavage in the carbon chain adjacent to the methoxy group [39].

\subsection{Principal component analysis}

Because of the large number of reference samples analysed, statistical treatment of data using PCA proved valuable to 

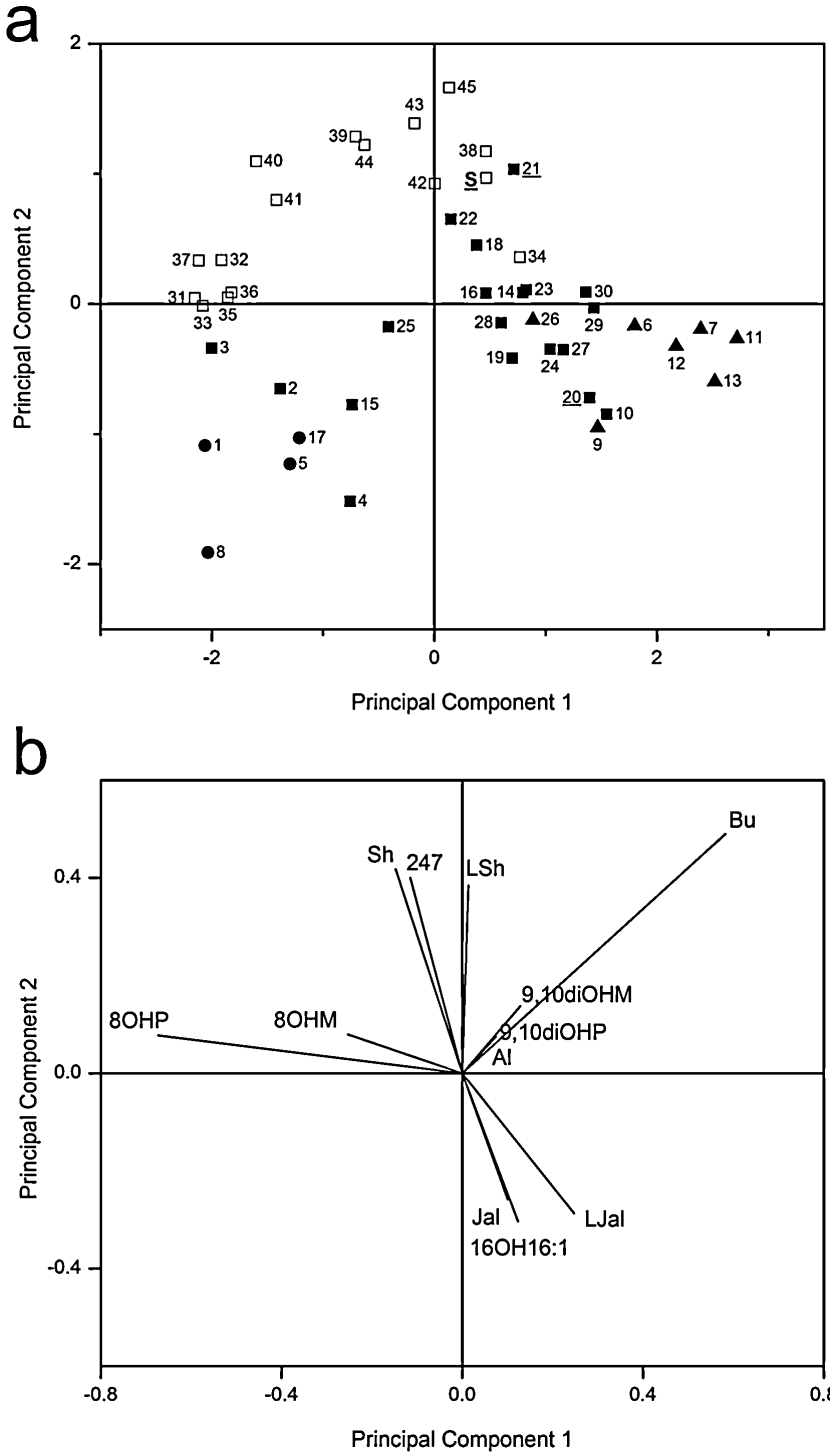

Fig. 3. PCA scores (a) and loadings (b) plots of THM-Py-GCMS data. Numbers in (a) refer to samples in Table 1; "S" is a varnish sample from the Signorelli painting. Solid figures are unbleached resins and open figures bleached resins. Triangles are resins of known Indian origin, circles are those of known Thai origin. For underlined scores see discussion in text.

visualise trends with regard to the composition of the resins that were not immediately apparent from visual comparison of the chromatograms. PC plots for the THM-Py-GCMS data are shown in Fig. 3, with scores (3a) numbered according to the samples in Table 1 and loadings ( $3 \mathrm{~b}$ ) corresponding to the chromatographic peaks used for the analysis (see Section 2 for details). Fatty alcohol derivatives were excluded from the calculation in order to provide information specifically on the resin component. The first two PCs are plotted, accounting for $73 \%$ of the variance of the dataset.

The distribution of scores and loadings can be interpreted in relation to the hydroxyacid composition of the resins. The samples in the upper half of Fig. 3a (with a positive score in the second PC) are mainly bleached shellacs, along with a few aged/historic samples, whereas most of those falling in the lower half are unbleached and recently acquired samples from artists' or industrial suppliers. This can be related to the loadings that contribute most strongly to the second PC: the grouping in the lower half of the loadings plot, Fig. 3b, includes jalaric (Jal), laccijalaric (LJal) and 16-hydroxyhexadecenoic (16OH16:1) acids. These compounds are reactive and prone to oxidation because of the presence of aldehyde (Jal, LJal) or alkene (16OH16:1) groups in their structures, and thus would be expected to diminish as a consequence of ageing or bleaching. A grouping of loadings in the opposing direction, in the upper half of the plot, includes shellolic (Sh) and laccishellolic (LSh) acids: these compounds contain a second carboxylic acid group that can be formed from the oxidation of the aldehyde group in jalaric and laccijalaric acids, respectively, and the PCA results support the idea that elevated levels of the former are a useful indicator for the aged or bleached resins $[6,46]$. Also in this group of loadings is an unidentified compound labelled " 247 ", from the base peak in its mass spectrum, that was included in the analysis because it has been interpreted by other researchers as a marker for aged shellacs $[47,48]$, an interpretation that is consistent with its position in the plot.

A useful illustration of this trend is provided by two samples of orange shellac from the historic Gettens Materials Collection (housed at the Straus Center for Conservation, Harvard Art Museums), one of which was taken from the bulk material (sample 20, data shown in Fig. 2) and the other from a film that was cast from alcohol solution onto a glass plate in 1932 (sample 21). The bulk sample occurs in the lower half of the plot in Fig. 3a and the sample from the cast film in the upper half, at a considerable separation, consistent with the advanced oxidation that would be expected in a thin film after c. 80 years.

The spread of samples in the horizontal axis (corresponding to the first PC) is associated mainly with the composition of aliphatic hydroxy acids, as indicated by the loadings for 8-hydroxy palmitic (8OHP) and 8-hydroxy myristic (8OHM) acids on the left, and 6-hydroxy myristic (butolic, Bu), 9,10-dihydroxy myristic (9,10diOHM), 9,10-dihydroxy palmitic (9,10diOHP) and 9,10,16trihydroxy palmitic (aleuritic, $\mathrm{Al}$ ) acids on the right, with the greatest variance associated with 8-hydroxy palmitic and butolic acids. The relative abundance of these components appears to be related to the biological origin of the samples: although most commercial suppliers of lac resins do not specify the source of their products, the examples that have a defined source fall into two groups, with Thai resins (samples 1, 5, 8 and 17) in the lower left quadrant, and Indian resins (samples $6,7,9,11,12,13,26$ ) in the lower right. An example of TMAH data for a Thai resin, showing prominent peaks for 8-hydroxy myristic and 8-hydroxy palmitic acids, and no observable peak for butolic acid, is shown in Fig. 2a. This finding is consistent with a previous nuclear magnetic resonance study that reported the occurrence of 8-hydroxy acids in resin produced by $K$. chinensis, the dominant species of lac insect in Thailand [49]. Wang et al. achieved a discrimination of a small sample set of Thai and Indian shellacs using THM-Py-GCMS with PCA, although their study did not report the 8-hydroxy compounds [20]. That these compounds, which appear to be valuable diagnostic markers, have not been reported in the chromatography literature is likely attributable in part to the fact that most previous research has been carried out on Indian resins. Note that in Fig. 3a the groups of Thai and Indian samples are centred below the positions of the loadings for the aliphatic hydroxyacids in 3b: this is because all of the resins of defined origin were recently acquired and unbleached samples, and the groups are thus weighted towards the lower half of the plot, associated with resins showing a lesser degree of oxidation. Analysis of a larger number of carefully sourced and provenanced raw materials will be valuable to confirm trends in relation to the hydroxyacid composition, since this could potentially provide useful contextual information on sources and trade of materials used in works of art.

PCA was also performed on data from GCMS analysis using TMTFTH of a subset of reference materials, using integrated areas of the same chromatographic peaks as for the TMAH data, and a similar distribution of scores and loadings was obtained (data not shown). 


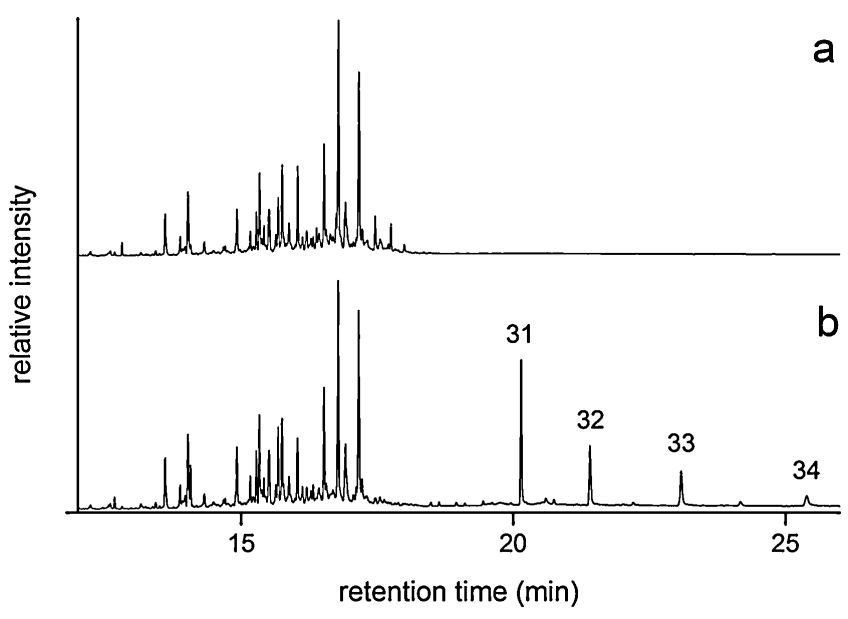

Fig. 4. Total ion chromatograms from THM-Py-GCMS analysis of an early twentiethcentury varnish, "Derayco Pure Shellac White", in the collection of the Florence Griswold Museum, Connecticut: (a) is from the translucent, upper phase of the varnish and (b) from the lower phase that is cloudy due to a high proportion of wax. Peak labels for wax components refer to Table 2.

The PCA analyses did not provide discernible groupings according to the various grades of unbleached shellac tested (lemon, orange, garnet, etc.). Although the use of additional chromatographic peaks and different data pre-treatments may provide further discrimination, this outcome suggests that the chemistry of the resin component of the shellacs is fundamentally similar and that these grades differ mainly in their residual dye content (not measured by GCMS).

\subsection{Further compositional differences: refining and bleaching}

Additional, qualitative differences in composition could be attributed to treatments used to produce certain grades of resin, namely refining and bleaching.

As mentioned in Section 1.1, shellac can be produced in wax-containing or wax-free grades, according to whether or not the naturally occurring lac wax is removed by filtration during processing. The de-waxed material is sometimes referred to as "refined" shellac [50]. Because of their characteristic pattern and separation from other compounds in the TIC the fatty alcohols (detected as methyl ethers using TMAH or TFMP ethers using TMTFTH) provide a clear indication of an unrefined (i.e. waxcontaining) grade of the resin. It should be noted that, in the case of shellac varnishes, the absence of significant levels of fatty alcohols in an unknown sample does not necessarily indicate a refined grade of resin, since the wax component can also be lost during the preparation (dissolution) of a varnish or as a result of its settling in a varnish solution that has been left to stand (Fig. 4).

Bleached shellac, introduced in the early nineteenth century, is produced by treatment of shellac or seedlac with chlorinebased bleaching agents, most commonly sodium hypochlorite, to remove colour associated with residual lac dye [22]. Although the major identified components were similar in the bleached and unbleached reference samples, increased levels of shellolic and laccishellolic acids were observed in bleached resins, associated with the oxidative effects of bleaching, as discussed above. More significantly, bleached shellacs could be differentiated qualitatively by the detection of trace levels of chlorinated compounds, discernible from characteristic chlorine $(\mathrm{Cl})$ isotope peak ratios in their mass spectra. An example is shown in Fig. 5, in which mass pairs with characteristic $3: 1 \mathrm{Cl}$ isotope ratios are present at $\mathrm{m} / \mathrm{z}$ $296 / 298,309 / 311,325 / 327,341 / 343$ and 356/358. AMDIS software was found to be a valuable tool for the detection of the markers

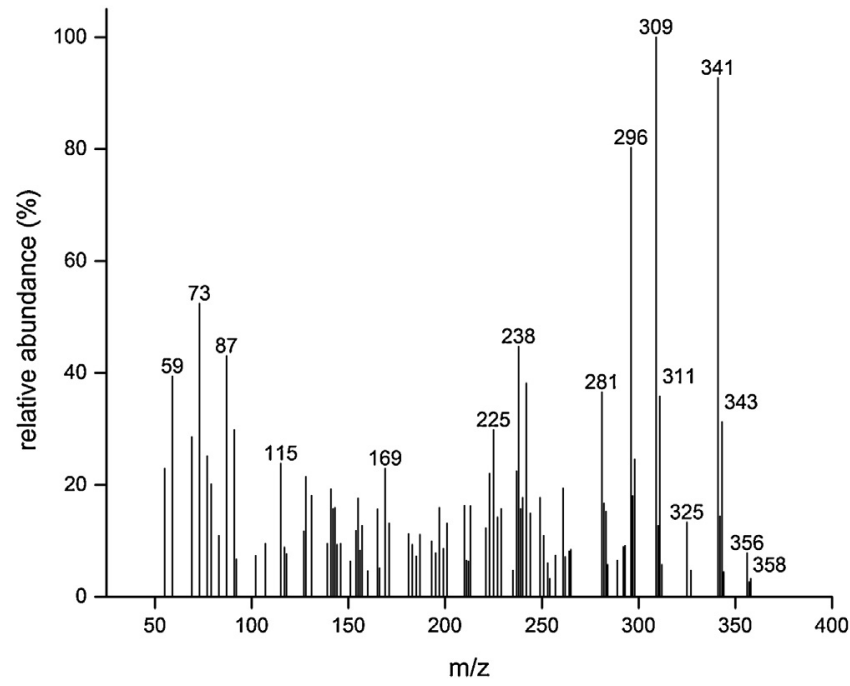

Fig. 5. EI mass spectrum for unidentified, chlorinated compound obtained from THM-Py-GCMS analysis of bleached shellac.

at very low levels. The presence of these compounds is consistent with studies of bleached shellac that have established that a small amount of $\mathrm{Cl}$ is incorporated into the resin as a consequence of bleaching with hypochlorite [7,16,51]. Research at the PMA dealing with the characterisation of the chlorinated marker compounds and their use for the identification of bleached shellac has been described in detail in previous publications $[21,22]$.

\subsection{Case study 1: Signorelli varnish}

During the recent conservation at the PMA of a fifteenthcentury painting by Luca Signorelli, The Annunciation (John G. Johnson collection, catalogue number 136), samples of varnish from the painting's surface were analysed to understand its treatment history and to inform the safe removal of the discoloured and disfiguring coatings. THM-Py-GCMS analysis revealed a composition of hydroxy aliphatic and terpene acids similar to those found in lac reference samples (Fig. 6). Prominent peaks in the TIC for derivatives of shellolic and laccishellolic acids are indicative of an aged and/or bleached resin (see also Fig. 3a in which the score for the varnish sample, labelled " $S$ ", is grouped with the other bleached and aged resins in the upper half of the PC plot). Upon close examination of the data, trace levels of chlorinated compounds characteristic of bleached shellac were discerned, confirming a varnish based on the bleached resin [22]. Fatty alcohols were detected (as their methyl ethers), indicating that the bleached shellac is a wax-containing (i.e. unrefined) grade. Also detected by THM-Py-GCMS were minor amounts of triterpenes characteristic of the plant resin dammar (methyl derivatives of dammarenolic acid and related compounds), fatty acids (primarily methyl esters of palmitic, stearic and the dicarboxylic azelaic acid) from drying oil, and a prominent peak for $n$-butyl methacrylate ( $n$ BMA). The complex composition of the samples, including shellac, dammar and acrylic resins, suggests that the painting has undergone several campaigns of varnishing. The fatty acids might indicate an oil component in the varnish, or alternatively could have migrated into the varnish from the underlying paint layers. The detection of the acrylic resin is significant since it highlights the importance of performing THM at high (pyrolytic) temperature, to allow the detection of a broad range of materials including those that are more readily analysed by conventional pyrolysis.

Research at the PMA has suggested that bleached shellac, or "white lac", experienced a period of popularity from the nineteenth 


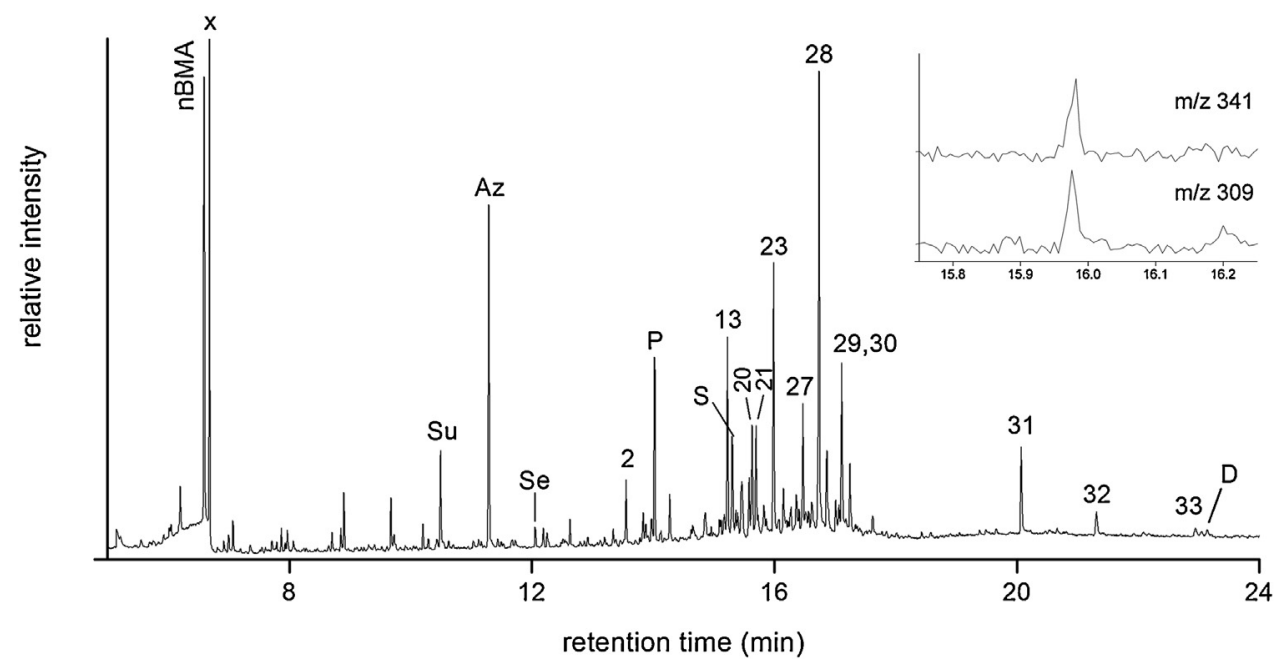

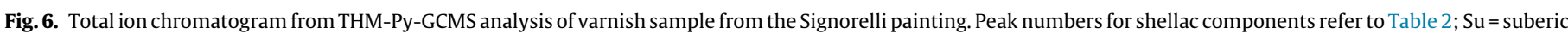

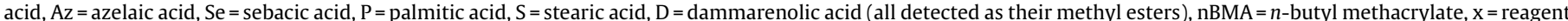
artefact. Inset: selected ion chromatogram details for $m / z 309$ and 341 indicating chlorinated marker for bleached shellac.

into the early twentieth century, both by artists and, in this case, as a restoration varnish [22]. The bleached resin is known to develop problems such as insolubility with age, however, likely related to the presence of residual chlorine [52]. Characterisation of the Signorelli varnish was therefore important to interpret its behaviour during treatment and to inform decisions about appropriate cleaning methods.

\subsection{Case study 2: inlay material on Dyer Chest}

A High Chest in the collection of the PMA by the Pennsylvania furniture maker George Dyer (a.k.a. Deyer; catalogue number 2009$1-1$ ), dated 1808 , features two inlaid designs depicting eagles that are characteristic of the Dyer family workshop (Fig. 7) [53]. Decorative inlays in nineteenth-century American furniture have not been widely studied (aside from some research on the sulfur inlay technique [54]) and samples from the Dyer Chest were analysed to provide insights into the materials used.

GCMS analysis of samples of black and white inlay materials using TMTFTH indicated that the organic component was beeswax (based on the detection of characteristic patterns of fatty acids, fatty alcohols and hydrocarbons, as described elsewhere [35]). Data for a sample of the red inlay, in contrast, indicated a material based on shellac, as shown in Fig. 8. Characteristic hydroxy aliphatic and terpene acid derivatives are observed, including prominent peaks for methyl and TFMP derivatives of shellolic and laccishellolic acids, along with smaller peaks for aleuritic acid derivatives. No chlorinated compounds were detected to suggest a bleached resin, which is expected since bleaching processes for lac were not introduced until the 1820s, after the chest was made [22]. Also detected in the red sample were methyl derivatives of diterpene acids (including dehydroabietic and 7-oxo-dehydroabietic acids) indicating a Pinaceae resin, probably pine resin. The red pigment was identified as vermilion (mercuric sulfide; analysed with Raman and energy dispersive spectroscopy). This combination of shellac, diterpene resin and vermilion is typical of nineteenth-century formulations for "sealing wax" [55]. The use of such a material for inlays is not surprising since it would have desirable properties such as malleability and durability, and sealing wax has been described as an inlay material for contemporary artefacts such as scrimshaw (carvings of whale bone, tooth, etc. [56]). To the best of authors' knowledge this is its first confirmed use in American furniture.
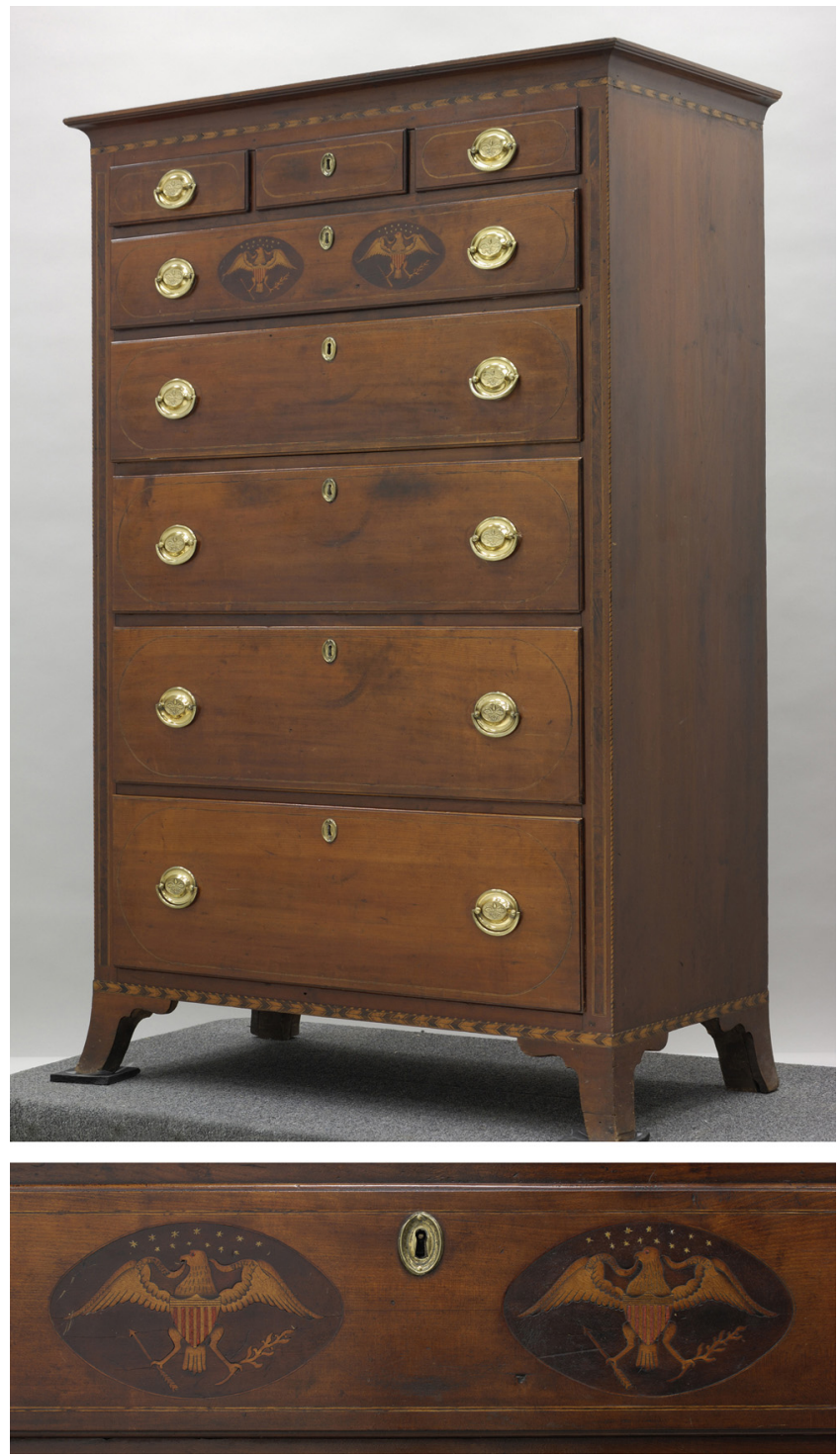

Fig. 7. George Dyer, High Chest, 1808, PMA Cat. 2009-1-1, with detail showing inlaid design. 


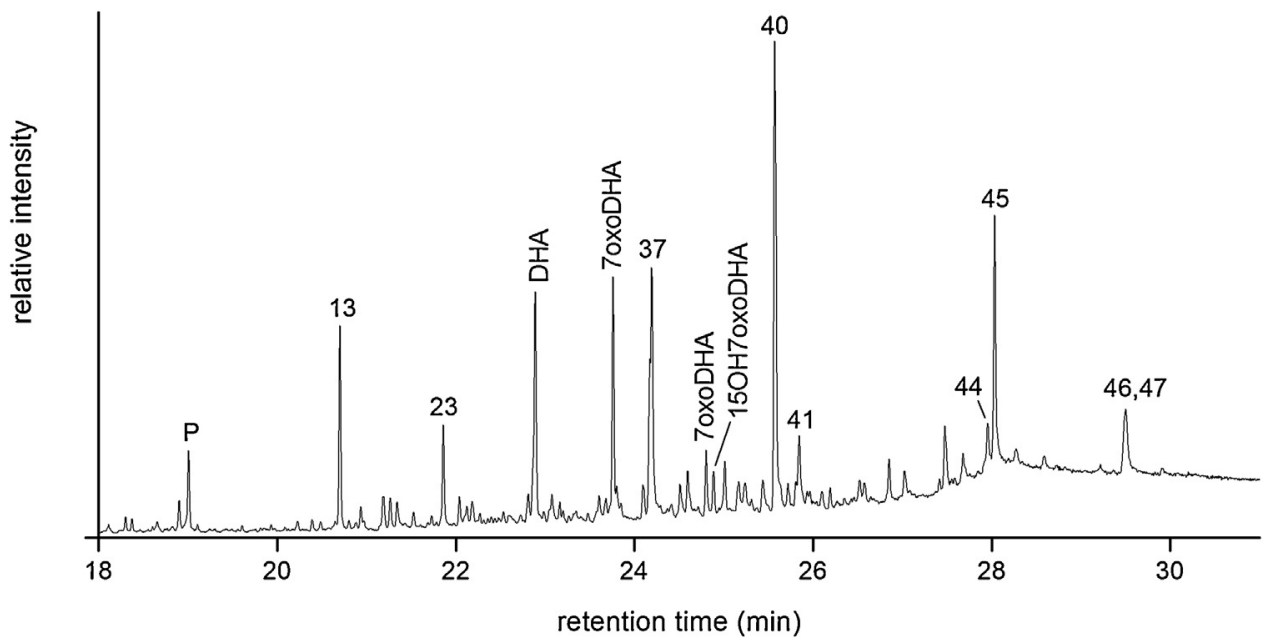

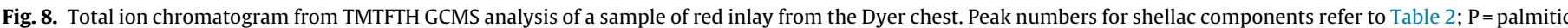

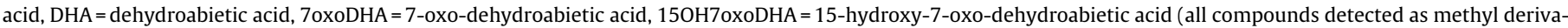
tives).

\subsection{Summary: strategies for data interpretation}

From this research, several key points can be summarised with regard to a refined strategy for the identification and discrimination of different types of lac resin, based on the recognition of key marker compounds:

- Derivatives of shellolic and laccishellolic acids are typically the most prominent, in combination with those of aleuritic and other aliphatic hydroxyacids, when lac resin is detected in samples from works of art. These terpene acids become more abundant in aged resins as a result of oxidation of jalaric and laccijalaric acids, respectively.

- The composition of aliphatic hydroxyacids in lac resins is variable, dependant on factors such as the biological source of the resin. In particular, butolic acid, which has been cited as a key marker compound for shellac $[19,20,47]$, may not always be present at detectable levels, and related compounds such as 8-hydroxy myristic and 8-hydroxy palmitic acids should be searched for as well.

- Bleached shellacs, in common with aged resins, show elevated levels of shellolic and laccishellolic acids as a consequence of the oxidising effect of bleaching. They can be identified more definitively from the detection of trace levels of chlorinated compounds, present in the resin as artefacts of the bleaching process $[21,22]$.

- Wax-containing (unrefined) lac resins can be discerned readily from the presence of a series of fatty alcohols (detected as their methyl ethers using TMAH, or TFMP ethers using TMTFTH) that elute towards the end of the chromatographic run.

\section{Conclusions}

This study demonstrates that GCMS and Py-GCMS using quaternary ammonium reagents (TMTFTH and TMAH, respectively) are powerful and sensitive methods for the identification of lac resin in aged microsamples from museum objects, requiring minimal sample preparation and allowing the separation and detection of characteristic hydroxy aliphatic and terpene acids that make up the resin. Several novel hydroxyacid derivatives are reported here for the first time as diagnostic components of lac, and previously unpublished mass spectra are included as an Appendix. Despite the complexity of the data, resulting from multiple derivatives of hydroxy compounds - particularly when using TMTFTH - both methods produce characteristic compounds that are effective as markers for the unambiguous identification of lac resin in unknown samples. Furthermore, from the study of a variety of reference samples, differences were observed according to their age, pretreatment (bleaching or refining), and biological origin, based on qualitative and quantitative variations in the chemical composition. This discrimination was enhanced by the use of principal component analysis. Further statistical studies of this type, taking into account minor hydroxyacids and other components, may be valuable to give a more precise differentiation of different types of lac resin.

One advantage of THM-Py-GCMS is that additional components in an unknown sample that would not be detectable using conventional GCMS, such as the acrylic in the Signorelli varnish, are identifiable from their pyrolysis products.

The analytical approaches have been applied successfully at the Philadelphia Museum of Art, illustrated here by the identification of a bleached shellac restoration varnish on a painting by Luca Signorelli, and a red inlay material in an early nineteenth-century High Chest by George Dyer that was made using a shellac-based "sealing wax". Such studies provide insights into historical materials and methods of manufacture of museum objects, shed light on their condition, and help to inform the development of appropriate conservation treatments.

\section{Acknowledgements}

KS is grateful to Beth Price, Andrew Lins, Allen Kosanovich, Mark Tucker and Peggy Olley at the PMA, and Joe Mikuliak for photography of the Dyer Chest. The following provided invaluable input on data interpretation and other aspects of the research: Ilaria Bonaduce, University of Pisa; Klaas Jan van den Berg, Netherlands Cultural Heritage Agency; Yasuyuki Ishida, Chubu University; Donald Dahlberg, Lebanon Valley College; Chris Petersen, Winterthur Museum; Tianlan Zhang, Dow Chemical Company; Glenn Frysinger and Greg Hall, United States Coast Guard Academy; Nobuko Shibayama, The Metropolitan Museum of Art. Reference samples were generously provided by Stephen Santos, Mantrose-Haeuser Company; Amy Kurtz Lansing, Florence Griswold Museum; Ursula Baumer, Doerner Institute; Narayan Khandekar, Harvard Art Museums; Melvin Wachowiak, Museum Conservation Institute, Smithsonian Institution; Tim Bryant, Temuss Products Ltd.; Paul Humphrey, W. S. Jenkins Co. Ltd; Gifu Shellac Manufacturing Co. Ltd. 


\section{Appendix.}

EI mass spectra of selected diagnostic compounds obtained from lac resin with derivatisation using TMAH and TMTFTH. Most spectra were deconvoluted using AMDIS software. Numbers correspond to Table 2.

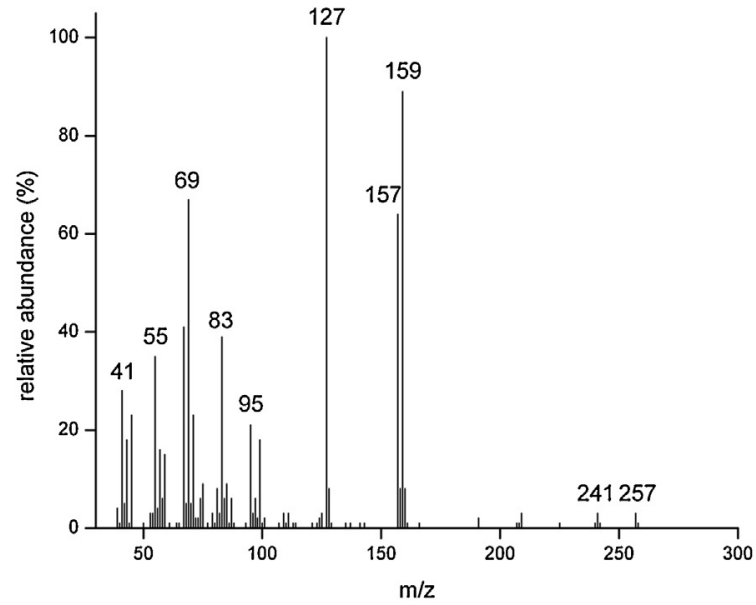

2. Butolic acid: methyl ester, methyl ether

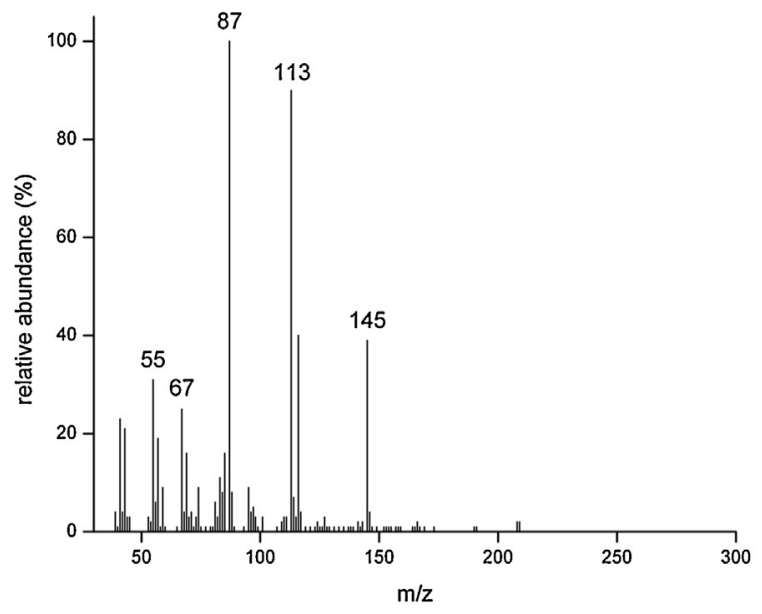

5. Butolic acid: methyl ester

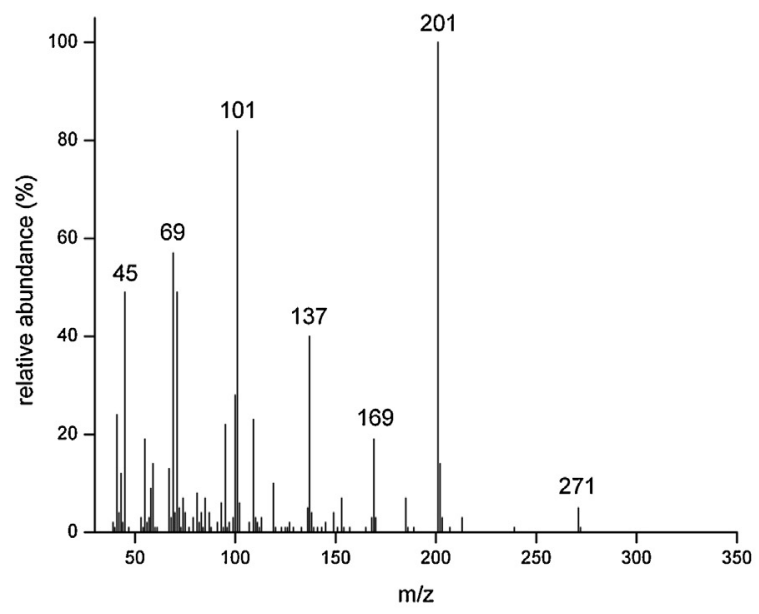

8. 9,10-dihydroxymyristic acid: methyl ester, dimethyl ether

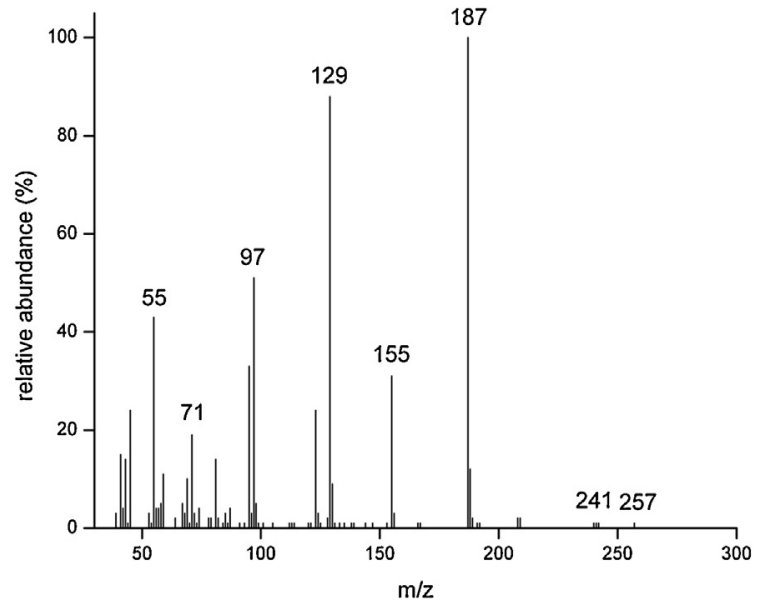

3. 8-hydroxymyristic acid: methyl ester, methyl ether

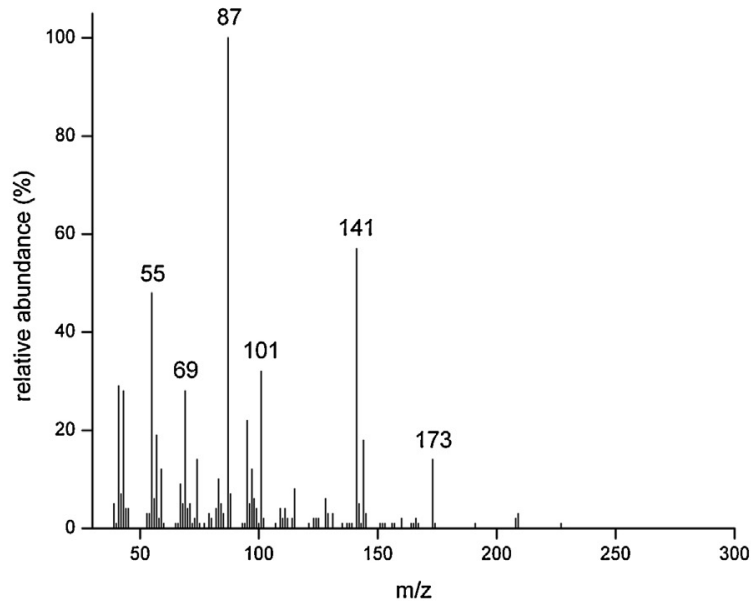

6. 8-hydroxymyristic acid: methyl ester

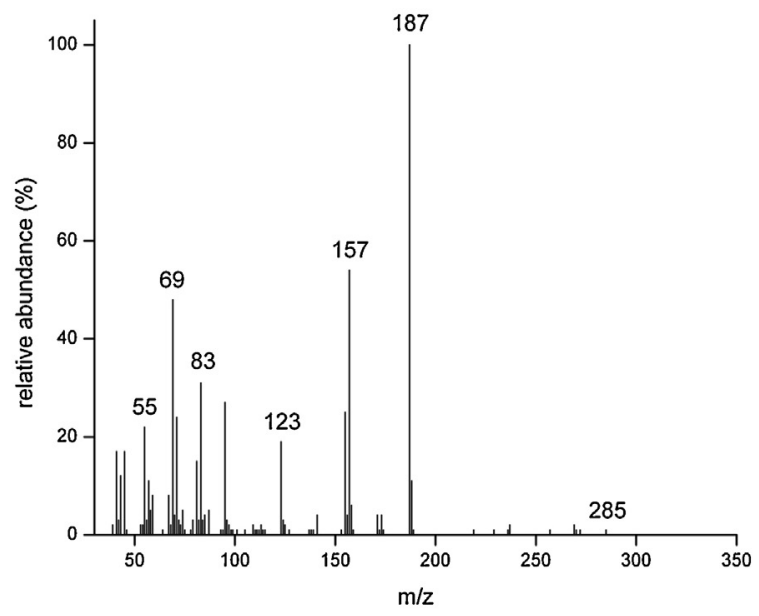

11. 8-hydroxypalmitic acid: methyl ester, methyl ether 


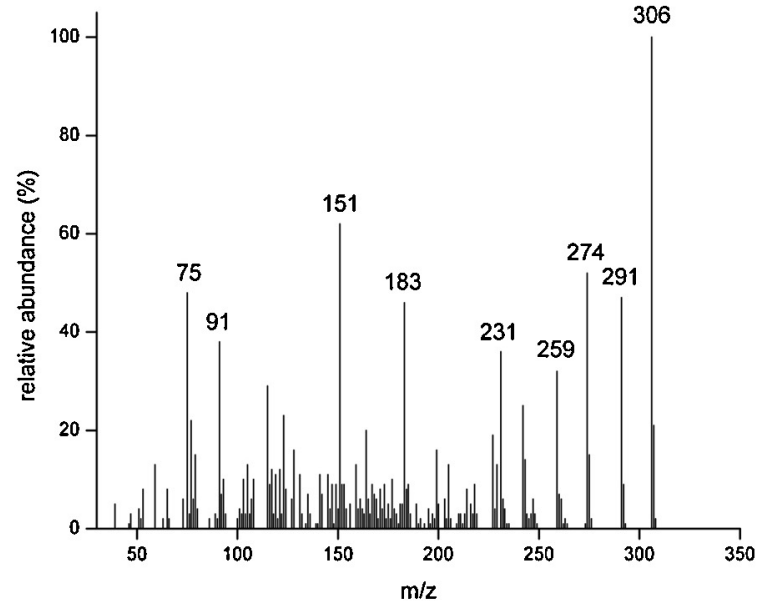

12. Laccijalaric acid: trimethyl derivative

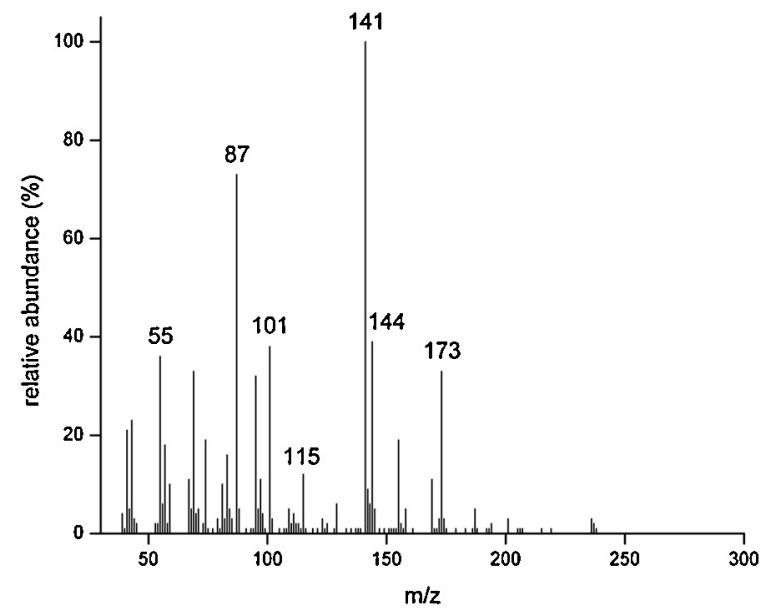

14. 8-hydroxypalmitic acid: methyl ester

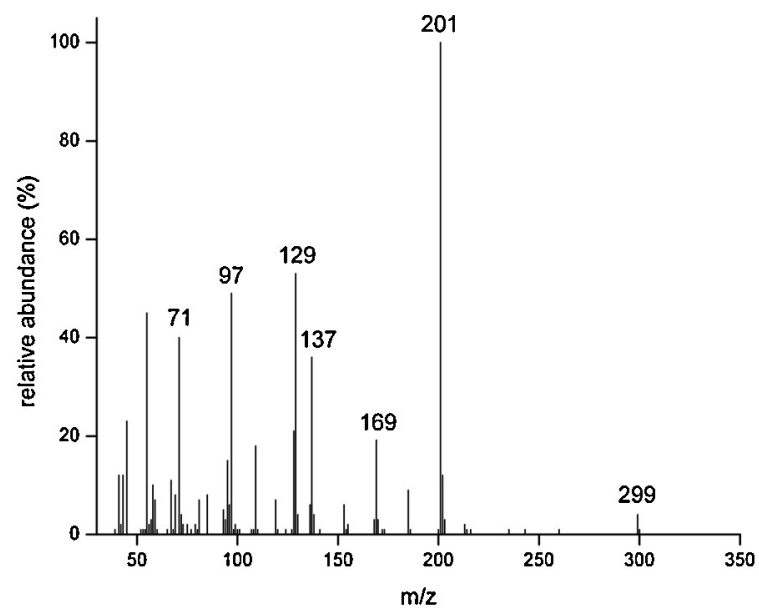

16. 9,10-dihydroxypalmitic acid: methyl ester, dimethyl ether

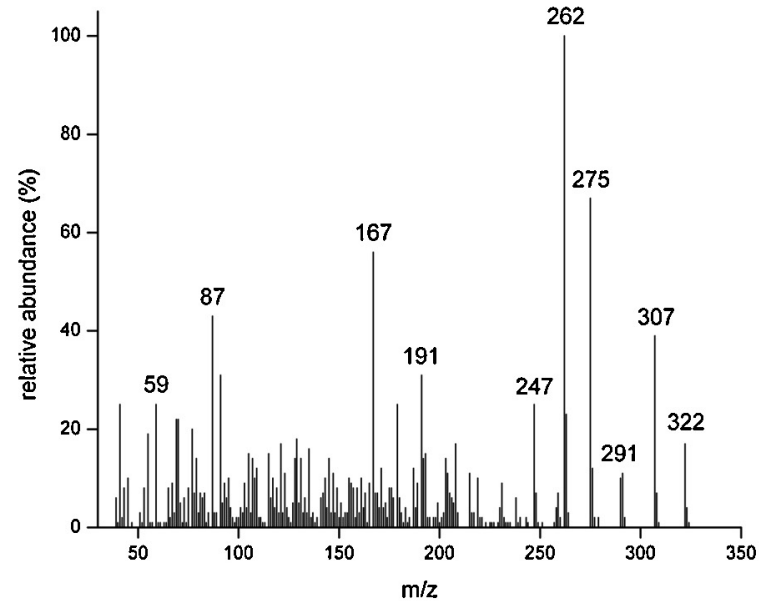

13. Laccishellolic acid: dimethyl ester, methyl ether

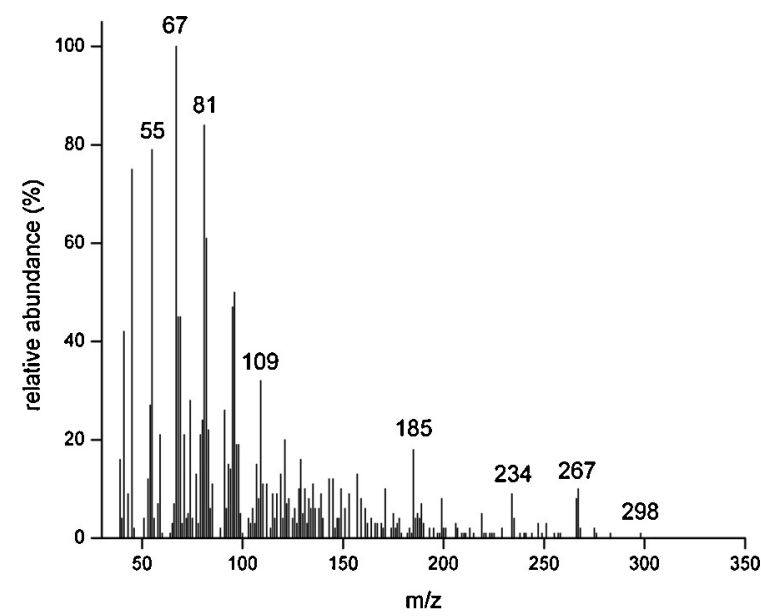

15. 16-hydroxyhexadecenoic acid: methyl ester, methyl ether

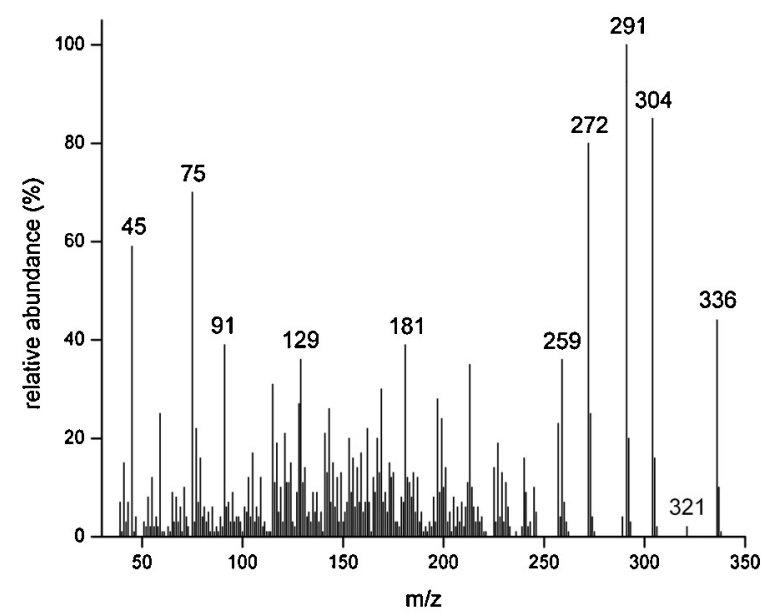

22. Jalaric acid: tetramethyl derivative 


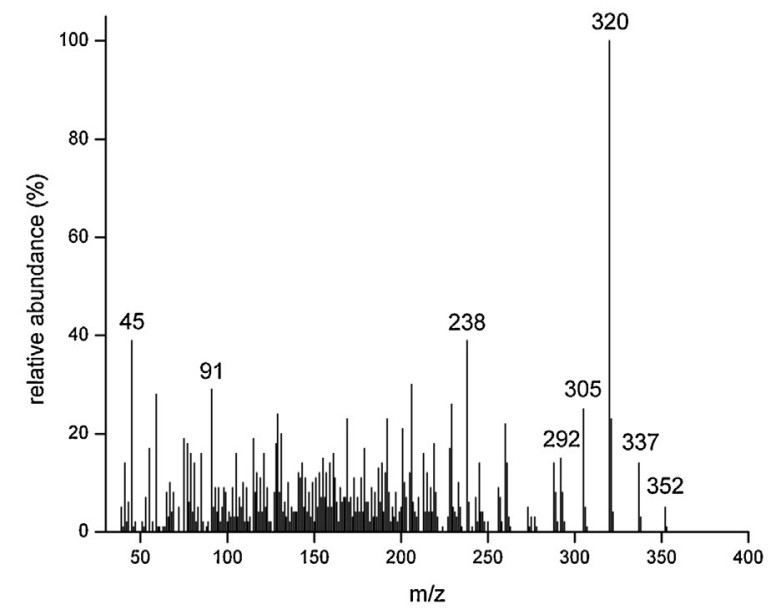

23. Shellolic acid: dimethyl ester, dimethyl ether

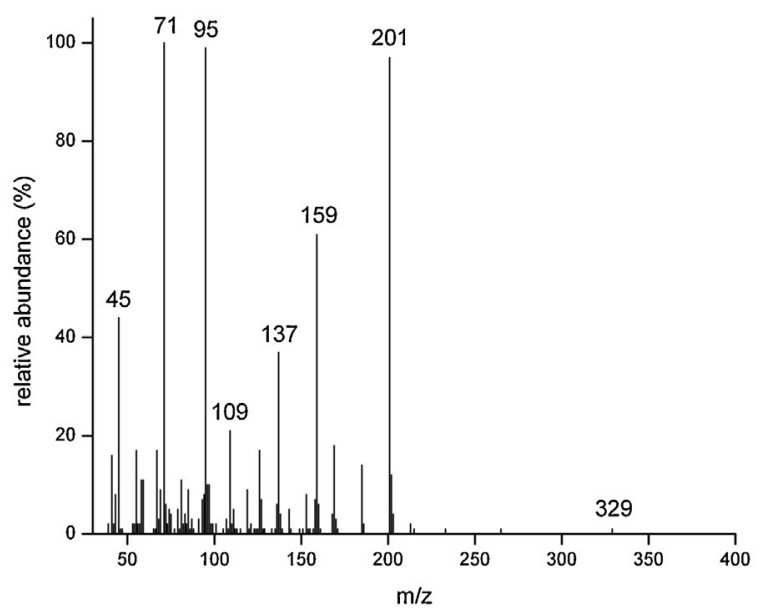

28. Aleuritic acid: methyl ester, trimethyl ether

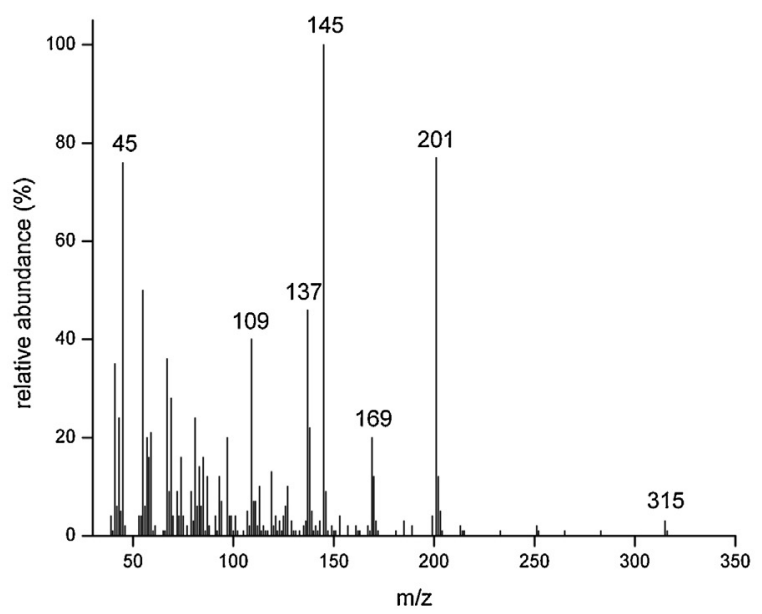

30. Aleuritic acid: methyl ester, 9,16-dimethyl ether

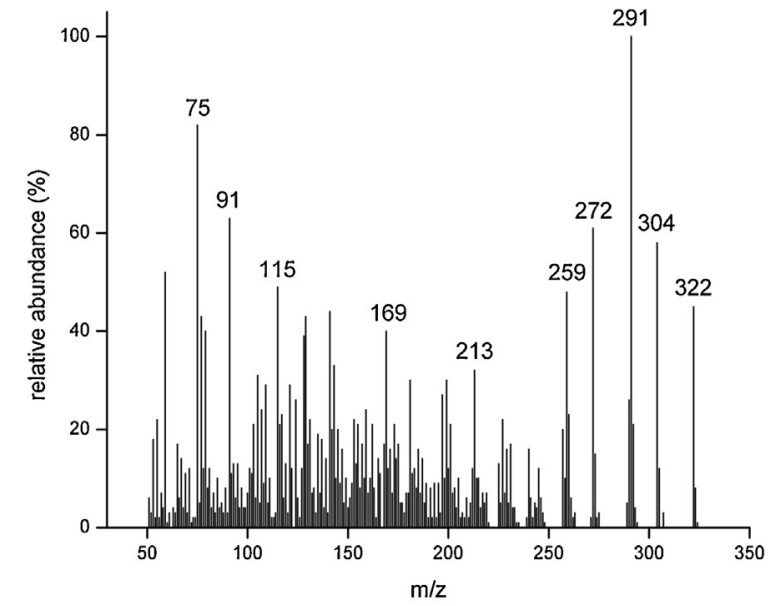

26. Jalaric acid: trimethyl derivative

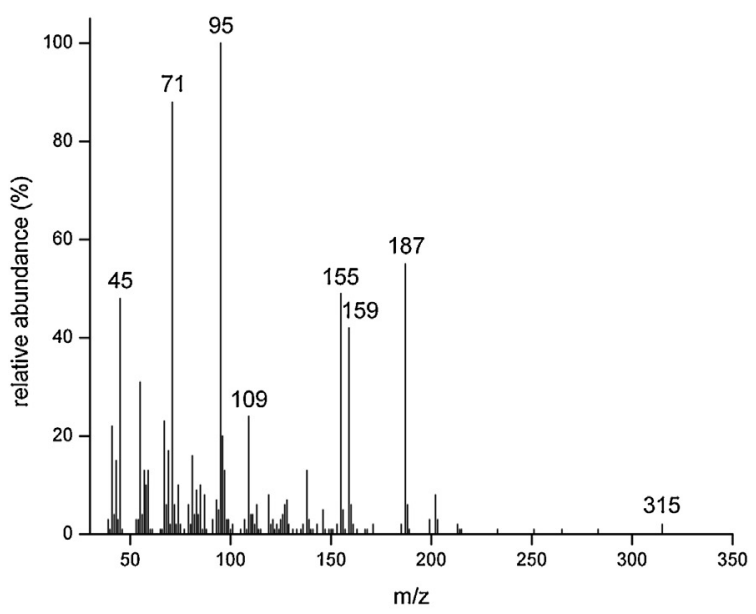

29. Aleuritic acid: methyl ester, 10,16-dimethyl ether

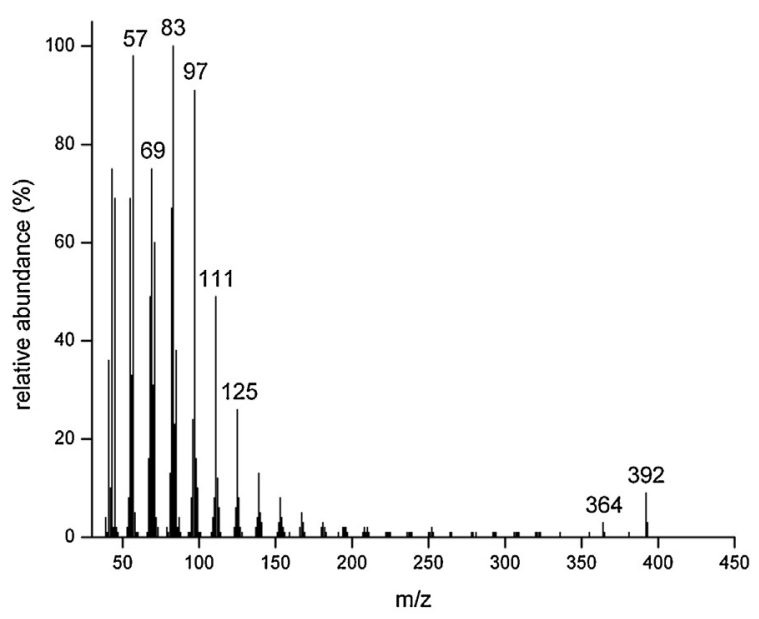

31. 1-octacosanol: methyl ether 


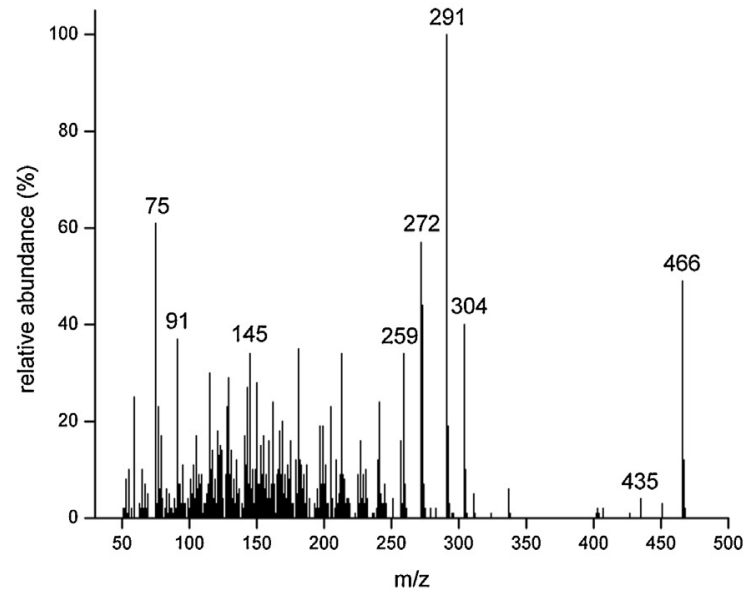

39. Jalaric acid: trimethyl, TFMP ether

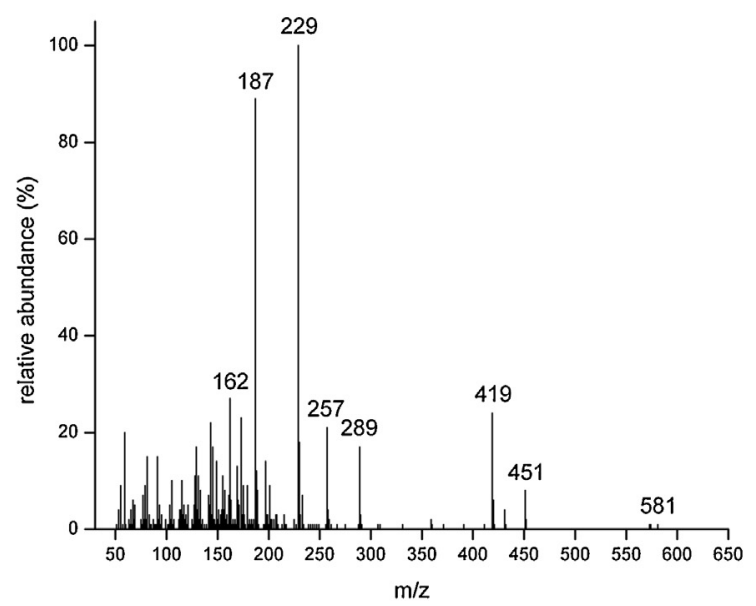

45. Shellolic acid: dimethyl ester, di-TFMP ether

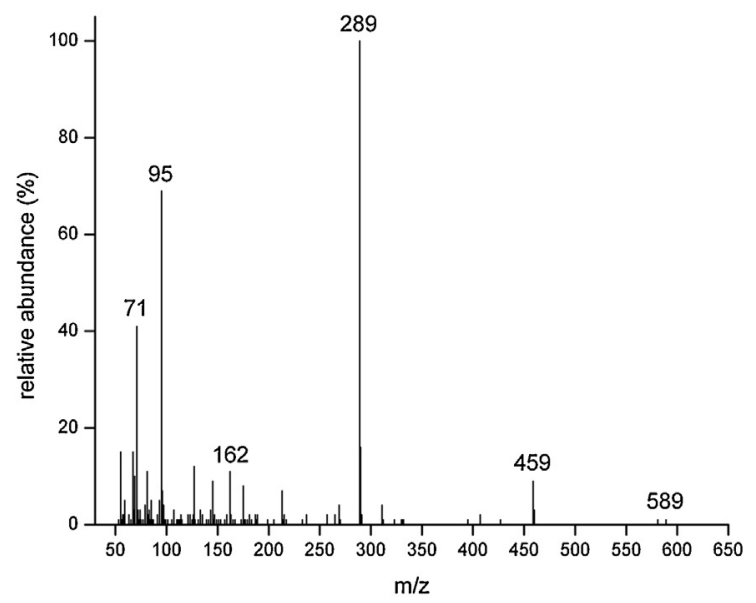

47. Aleuritic acid: methyl ester, 10-methyl ether, 9,16-di-TFMP ether

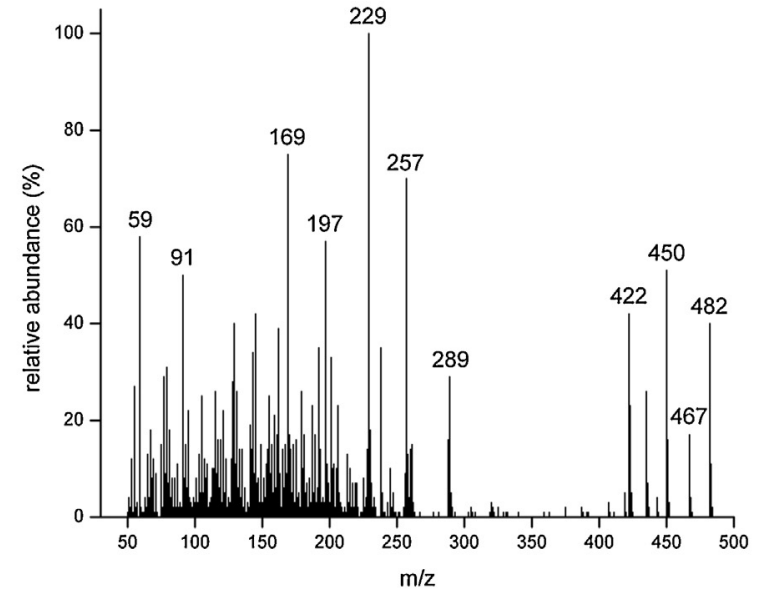

40. Shellolic acid: dimethyl ester, methyl ether, TFMP ether

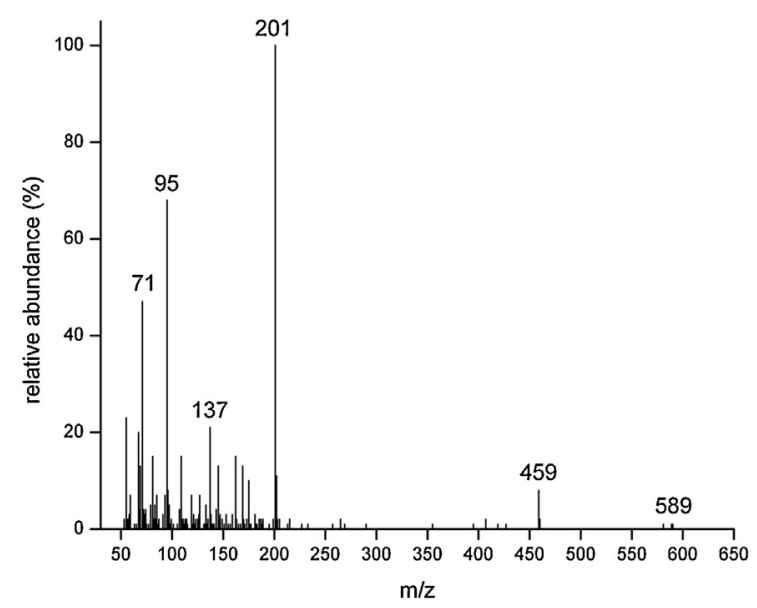

46. Aleuritic acid: methyl ester, 9-methyl ether, 10,16-di-TFMP ether

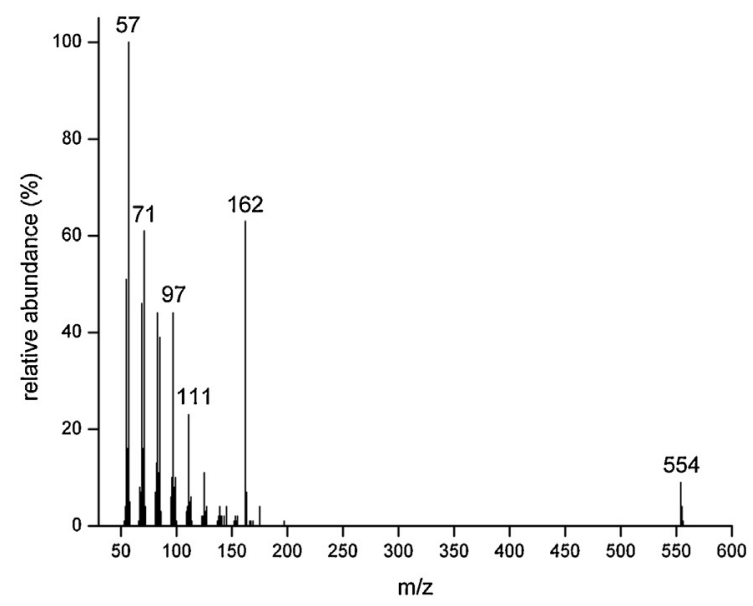

49. 1-octacosanol: TFMP ether 


\section{References}

[1] D. Cardon, Natural Dyes: Sources, Tradition, Technology and Science, Archetype Publications Ltd., London, 2007, p. 656.

[2] S.K. Ranjan, C.B. Mallick, D. Saha, A.S. Vidyarthi, R. Ramani, Genet. Mol. Biol. 34 (2011) 511.

[3] X. Chen, H. Chen, Y. Feng, R. He, Z. Yang, J. Insect Sci. 11 (106) (2011).

[4] A.C. Langmuir, in: A. Rogers (Ed.), Industrial Chemistry: A Manual for the Student and Manufacturer, 2nd ed., D. Van Nostrand Company, New York, 1915, p. 696.

[5] J.W. Martin, in: R.R. Myers, J.S. Long (Eds.), Treatise on Coatings Film-Forming Compositions, Part III, 1, Marcel Dekker, New York, 1972, p. 441.

[6] J.S. Mills, R. White, The Organic Chemistry of Museum Objects, 2nd ed. Butterworth-Heinemann, Oxford, 1994, pp. 115, 180

[7] S.A. Santos, J.F., Cotter, M.M. McWeeney, US Patent 6,348,217 (19 February 2002).

[8] K.S. Brown, Chem. Soc. Rev. 4 (1975) 263.

[9] A.N. Singh, A.B. Upadhye, V.V. Mhaskar, S. Dev, Tetrahedron 30 (1974) 3689.

[10] M. Derrick, J. Am. Inst. Conserv. 28 (1989) 43.

[11] S. Prati, G. Sciutto, R. Mazzeo, C. Torri, D. Fabbri, Anal. Bioanal. Chem. 399 (2011) 3081.

[12] C. Daher, C. Paris, A.-S. Le Hô, L. Bellot-Gurlet, J.P. Échard, J. Raman Spectrosc. $41(2010) 1494$

[13] A. Nevin, D. Comelli, G. Valentini, R. Cubeddu, Anal. Chem. 81 (2009) 1784.

[14] A. Nevin, J.P. Echard, M. Thoury, D. Comelli, G. Valentini, R. Cubeddu, Talanta 80 (2009) 286.

[15] J.S. Mills, R. White, The Organic Chemistry of Museum Objects, 2nd ed. Butterworth-Heinemann, Oxford, 1994, p. 20.

[16] K. Buch, M. Penning, E. Wächtersbach, M. Maskos, P. Langguth, Drug Dev. Ind Pharm. 35 (2009) 694

[17] P. Dietemann, C. Herm, in: M.P. Colombini, F. Modugno (Eds.), Organic Mass Spectrometry in Art and Archaeology, John Wiley \& Sons, Ltd., Chichester, 2009 p. 147.

[18] G. Chiavari, D. Fabbri, R. Mazzeo, P. Bocchini, G.C. Galletti, Chromatographia 41 (1995) 273.

[19] M.P. Colombini, I. Bonaduce, G. Gautier, Chromatographia 58 (2003) 357.

[20] L. Wang, Y. Ishida, H. Ohtani, S. Tsuge, Anal. Chem. 71 (1999) 1316.

[21] K. Sutherland, M. Szelewski, Mass Matters, 59th ed., British Mass Spectrometry Society, 2009, July, p. 15.

[22] K. Sutherland, J. Inst. Conserv. 33 (2010) 129

[23] W.C. Kossa, J. MacGee, S. Ramachandran, A.J. Webber, J. Chromatogr. Sci. 17 (1979) 177

[24] J.M. Challinor, J. Anal. Appl. Pyrol. 37 (1996) 185

[25] J.M. Challinor, J. Anal. Appl. Pyrol. 61 (2001) 3.

[26] I. Pastorova, K.J. van den Berg, J.J. Boon, J.W. Verhoeven, J. Anal. Appl. Pyrol. 43 (1997) 41
[27] H. van Keulen, Int. J. Mass Spectrom. 284 (2009) 162.

[28] S. Watts, E.R. de la Rie, Stud. Conserv. 47 (2002) 257

[29] R. White, J. Pilc, Natl. Gallery Tech. Bull. 17 (1996) 95.

[30] M.A. Fedrigo, M. Favaro, P. Traldi, Rapid Commun. Mass Spectrom. 14 (2000) 2203.

[31] V. Pitthard, P. Finch, T. Bayerová, J. Sep. Sci. 27 (2004) 200

[32] V. Pitthard, S. Stanek, M. Griesser, T. Muxeneder, Chromatographia 62 (2005) 175.

[33] J.W. de Leeuw, M. Baas, J. Anal. Appl. Pyrol. 26 (1993) 175.

[34] J. Carlson, W.C. Petersen, in: V. Dorge (Ed.), Solvent Gels for the Cleaning of Works of Art: The Residue Question, The Getty Conservation Institute, Los Angeles, 2004, p. 111

[35] K. Sutherland, J. Chromatogr. A 1149 (2007) 30.

[36] S. Wold, K. Esbensen, P. Geladi, Chemom. Intell. Lab. Syst. 2 (1987) 37.

[37] M.S. Wagner, D.J. Graham, B.D. Ratner, D.G. Castner, Surf. Sci. 570 (2004) 78

[38] P.G. Kralert, R. Alexander, R.I. Kagi, Org. Geochem. 23 (1995) 627.

[39] J.C. del Río, P.G. Hatcher, Org. Geochem. 29 (1998) 1441.

[40] I. Tanczos, M. Schöflinger, H. Schmidt, J. Balla, J. Anal. Appl. Pyrol. 42 (1997) 21.

[41] I. Tanczos, K. Rendl, H. Schmidt, J. Anal. Appl. Pyrol. 49 (1999) 319.

[42] C. Schwarzinger, J. Anal. Appl. Pyrol. 68-69 (2003) 137.

[43] S. Tsuge, H. Ohtani, C. Watanabe, Pyrolysis-GC/MS Data Book of Synthetic Polymers, Elsevier, Amsterdam, 2011, p. 380.

[44] J.D.J. van den Berg, J.J. Boon, J. Anal. Appl. Pyrol. 61 (2001) 45.

[45] E. Faurot-Bouchet, G. Michel, J. Am. Oil Chem. Soc. 41 (1964) 418.

[46] C. Coelho, R. Nanabala, M. Ménager, S. Commereuc, V. Verney, Polym. Degrad. Stabil. 97 (2012) 936

[47] A. Heginbotham, H. Khanjian, R. Rivenc, M. Schilling, 15th Triennial Conference New Delhi 22-26 September 2008, ICOM Committee for Conservation, Preprints Volume II, Allied Publishers Pvt. Ltd., New Delhi, 2008, p. 1100.

[48] H. van Keulen, Netherlands Cultural Heritage Agency, unpublished data.

[49] A. Ichikawa, H. Takahashi, T. Ooi, T. Kusumi, Biosci. Biotech. Biochem. 61 (1997) 881.

[50] E. Hicks, Shellac: Its Origin and Applications, Chemical Publishing Co., Inc., New York, 1961, p. 32

[51] B.B. Khanna, J. Appl. Chem. 20 (1970) 392.

[52] N.N. Murty, Ind. Eng. Chem. 31 (1939) 235.

[53] W.A. Cooper, Paint, Pattern \& People: Furniture of Southeastern Pennsylvania, 1725-1850, The Henry Francis du Pont Winterthur Museum Inc., Winterthur, DE, 2011, p. 183

[54] J.L. Mass, M.J. Anderson, Measure. Sci. Technol. 14 (2003) 1598.

[55] A.J. Cooley, The Book of Useful Knowledge: A Cyclopaedia of Six Thousand Practical Receipts and Collateral Information in the Arts, Manufactures, and Trades, D. Appleton \& Company, New York, 1854, p.560.

[56] G.C. Wertkin, L. Kogan, Encyclopedia of American Folk Art, Routledge, New York, 2004, p. 467. 